\title{
Equivariant Surgery Theory: Construction of Equivariant Normal Maps
}

\author{
Dedicated to Prof. S. Sasao on his sixtieth birthday
}

By

Masaharu MoRIMOTo*

§0. Introduction

A routine to construct exotic smooth actions is to proceed the following two:

(Step I) Construction of an equivariant normal map.

(Step II) Killing equivariant surgery obstructions.

This procedure is one of the important ideas of equivariant surgery theory, and has enabled us to construct various exotic actions (see e.g. [BMo1-2], [LaMo], [LaMoPa], [Mo1-3], [MoU], [Pe1-3], [PeR]). A method for (Step I) was presented by $\mathrm{T}$. Petrie in [Pe1-3], which we call the equivariant transversality construction. Roughly speaking, it is as follows: Let $G$ be a finite group, and let $Y$ be a compact, smooth $G$-manifold. If $V$ is a real $G$-module and $\alpha: Y \times V \rightarrow Y \times V$ is a proper $G$-map then $\alpha$ is properly $G$-homotopic to $\beta$ : $Y \times V \rightarrow Y \times V$ such that $\beta$ is transversal to $Y \times\{0\}$. Then we obtain a $G$-normal map $f: X \rightarrow Y$, where $X=\beta^{-1}(Y \times\{0\})$ and $f=\left.\beta\right|_{X}: X \rightarrow Y$. (Step II) is to convert $f: X \rightarrow Y$ to a $G$-map $f^{\prime}: X^{\prime} \rightarrow Y$ belonging to a prescribed class of maps, e.g. of $G$-homotopy equivalences, of homotopy equivalences, of $\mathbb{Z}_{p}$-homology equivalences, etc. If some properties of $X^{\prime}$ are specified before the construction then it is a key to find an adequate real $G$-module $V$ and an appropriate $G$-map $\alpha$. Modified equivariant transversality construction has been employed in [BMo1-2], [LaMoPa], [LaMo], [Mo1-3], [MoU]. For

Communicated by K. Saito, April 21, 1994.

1991 Mathematics Subject Classifications: 57R65, 57R85, 57R91.

Partially supported by Grant-in-Aid for Scientific Research.

* Department of Environmental and Mathematical Sciences, Faculty of Environmental Science and Technology, Okayama University, Okayama 700, Japan. 
further applications of equivariant surgery theory, in the present paper we give improvements in the equivariant transversality construction. In subsequent papers, we will discuss (Step II) and applications.

Main results of the present paper are Theorems 1.3, 2.7, 2.12, and 4.4, although Theorem 2.12 is an easy generalization of Laitinen's result [LaMo, Theorem 1.3]. Theorem 0.1 below follows from these. In the papers referred above, some details were omitted, for those were regarded as well-known facts. But the current paper contains the details, because the author has been sometimes asked to give the details (e.g. Proofs of Lemmas 4.6 and 4.7).

Throughout this paper let $G$ be a finite group. If $H$ is a subgroup of $G$ then we write $H \leqq G$. Let $\mathscr{S}(G)$ be the set of all subgroups of $G$. When we regard $\mathscr{S}(G)$ as a $G$-set, the action is one defined by $(g, H) \mapsto g H^{-1}(g \in G$ and $H \in \mathscr{S}(G)$ ). For a $G$-space $X$ let Iso $(G, X) \subseteq \mathscr{S}(G)$ be the set of all isotropy subgroups at points in $X$. Let $\mathscr{M}(G)(\subseteq \mathscr{S}(G) \backslash\{G\})$ denote the set of all proper subgroups $K$ never including a subgroup $H$ such that $H \triangleleft G$ and that $G / H$ is of prime power order (cf. (2.8)). We call $G$ an Oliver group if there does not exist a series of subgroups $P \triangleleft H \triangleleft G$ such that $P$ and $G / H$ are of prime power order and $H / P$ is a cyclic group. Let $\mathbb{R}$ (resp. $\mathbb{C}$ ) be the real (resp. complex) number field with trivial $G$-action. $\mathbb{C}[G]$ denotes the group ring of $G$ with coefficients in $\mathbb{C}$. This is canonically regarded as a complex (left) $G$-module of dimension $|G|$. Let $\mathbb{C}[G]^{m}$ be the $m$-fold direct sum of $\mathbb{C}[G]$ 's. If $V$ is a real $G$-module and $X$ is a $G$-space then let $\varepsilon_{X}(V)$ stand for the product bundle $X \times V$ over $X$ with fiber $V$. Adopting the notation, we have the next theorem.

Theorem 0.1. Let $G$ be an Oliver group, and let $Y$ be a compact, connected, oriented, smooth G-manifold with disjoint sub-G-simplicial complexes $Z_{1}, \cdots, Z_{l}$ (with respect to some equivariant smooth triangulation of $Y$ ). Suppose Iso $\left(G, Y \backslash \bigcup_{i=1}^{l} \mathrm{RN}\left(Z_{i}\right)\right) \subseteq \mathscr{M}(G)$, where $\mathrm{RN}\left(Z_{i}\right)$ 's are disjoint closed G-regular neighborhoods (being G-submanifolds of $Y$ ) of $Z_{i}$ 's, respectively. If $n_{1}, \ldots, n_{l}$ are nonnegative integers then there exists a G-normal map $(f, b)(c f . \S 3)$ (where $f:(X, \partial X) \rightarrow(Y, \partial Y)$ is a G-map, and $b: T(X) \oplus \varepsilon_{X}\left(\mathbb{C}[G]^{m}\right) \rightarrow f^{*} T(Y) \oplus \varepsilon_{X}\left(\mathbb{C}[G]^{m}\right)$ is a $G$-vector bundle isomorphism for some natural number $m$ ) satisfying the following (0.1.1)-(0.1.4):

(0.1.1) Let $\mathrm{RN}\left(Z_{i}^{G}\right)$ be a closed G-regular neighborhood of $Z_{i}^{G}$ in $\mathrm{RN}\left(Z_{i}\right)$. For every $i=1, \ldots, l, f^{-1}\left(\mathrm{RN}\left(Z_{i}^{G}\right)\right)$ is $G$-diffeomorphic to $\operatorname{RN}\left(Z_{i}^{G}\right)_{1} \cup \cdots \cup \operatorname{RN}\left(Z_{i}^{G}\right)_{n_{2}}$ $\left(n_{i}\right.$-fold disjoint union of copies of $\mathrm{RN}\left(Z_{i}^{G}\right)$ ).

(0.1.2) Each $\left.f\right|_{\mathrm{RN}\left(Z_{i}^{G}\right)_{j}}: \mathrm{RN}\left(Z_{i}^{G}\right)_{j} \rightarrow \mathrm{RN}\left(Z_{i}^{G}\right)$ is the canonical G-diffeomorphism.

(0.1.3) $\operatorname{deg}\left(f^{H}: X^{H} \rightarrow Y^{H}\right)=1$ whenever $H \in M(G)$ and $Y^{H}$ is connected and oriented.

(0.1.4) For each $H \in \mathscr{M}(G)$, there exists an $H$-normal cobordism 


$$
\left({ }_{H} F,{ }_{H} B\right):\left(\operatorname{Res}_{H}^{G} f, \operatorname{Res}_{H}^{G} b\right) \sim\left(i d_{\operatorname{Res}_{H}^{G} Y}, i d_{\operatorname{Res}_{H}^{G}\left(T(Y) \oplus \varepsilon_{Y}\left(\mathbb{C}[G]^{m}\right)\right)}\right)
$$

for sufficiently large $m$.

Proof. By Theorem 2.7, there exists $\alpha \in \Omega(G)$ (the Burnside ring of $G$ ) such that $\chi_{G}(\alpha)=1$ in the ring $\mathbb{Z}$ of integers and $\operatorname{Res}_{H}^{G}(\alpha)=0$ in $\Omega(H)$ for all $H \in \mathscr{M}(G)$. (The definition of $\chi_{G}(\quad)$ is given in $\S 1$.) Let $1_{\Omega}$ be the unity in $\Omega(G)$. Applying Theorem 1.3 for $\gamma_{i}=n_{i} 1_{\Omega}$, we obtain $\omega^{\prime} \in \omega_{G}^{0}(Y)$ such that $j_{\left(Y, Z_{i}\right)}^{*}\left(\omega^{\prime}\right)=\alpha^{s}\left(n_{i} 1_{\Omega}\right)_{Z_{i}}$ in $\omega_{G}^{0}\left(Z_{i}\right)$ for a suitable natural number $s$, where $j_{\left(Y, Z_{i}\right)}$ : $Z_{i} \rightarrow Y$ is the canonical inclusion and $\left(n_{i} 1_{\Omega}\right)_{Z_{i}}=p_{i}^{*}\left(n_{i} 1_{\Omega}\right)$ for $p_{i}: Z_{i} \rightarrow p t$ (see $\S 1$ for details of definition of $\left.\left(n_{i} 1_{\Omega}\right)_{Z_{\imath}}\right)$. Let $\omega=1_{Y}-\alpha\left(1_{Y}-\omega^{\prime}\right)$ and $\gamma_{i}=1_{\Omega}-$ $\alpha\left(1_{\Omega}-n_{i} \alpha^{s}\right)$. Further let $\varphi_{H}^{ \pm}\left(\gamma_{i}\right)((H) \in \mathscr{S}(G) / G)$ be nonnegative integers such that

$$
\gamma_{i}=\sum_{\boldsymbol{H}}\left(\varphi_{H}^{+}\left(\gamma_{i}\right)-\varphi_{\boldsymbol{H}}^{-}\left(\gamma_{i}\right)\right)[G / H]
$$

and that for each $H$, either $\varphi_{H}^{+}\left(\gamma_{i}\right)$ or $\varphi_{H}^{-}\left(\gamma_{i}\right)$ is equal to 0 . Let $\varphi_{H}\left(\gamma_{i}\right)=\varphi_{H}^{+}\left(\gamma_{i}\right)-$ $\varphi_{H}^{-}\left(\gamma_{i}\right)$. In this case, clearly $\left|\varphi_{H}\left(\gamma_{i}\right)\right|=\max \left(\varphi_{H}^{+}\left(\gamma_{i}\right), \varphi_{H}^{-}\left(\gamma_{i}\right)\right)$. Then, by Theorem 4.4 we get a $G$-normal map $(f, b)$ satisfying (4.4.1)-(4.4.3). By (4.4.1), forgetting orientations, $f^{-1}\left(\mathrm{RN}\left(Z_{i}^{G}\right)\right)$ is $G$-diffeomorphic to

$$
\mathrm{RN}\left(Z_{i}^{G}\right) \cup \cdots \cup \mathrm{RN}\left(Z_{i}^{G}\right)\left(\left(\left|\varphi_{G}^{+}\left(\gamma_{i}\right)\right|+\left|\varphi_{G}^{-}\left(\gamma_{i}\right)\right|\right)\right. \text {-fold disjoint union) . }
$$

It holds that $\varphi_{G}\left(\gamma_{i}\right)=\varphi_{G}\left(1_{\Omega}-\alpha\left(1_{\Omega}-n_{i} \alpha^{s}\right)\right)=1-\chi_{G}(\alpha)+n_{i} \chi_{G}(\alpha)^{s+1}$. Since $\chi_{G}(\alpha)=1$, we get $\varphi_{G}\left(\gamma_{i}\right)=n_{i}$. This implies that $\varphi_{G}^{+}\left(\gamma_{i}\right)=n_{i}$ and $\varphi_{G}^{-}\left(\gamma_{i}\right)=0$. Thus (0.1.1) and (0.1.2) immediately follow from (4.4.1) (in this case, $K_{i}=G$ ). Suppose that $H \in \mathscr{M}(G)$ and that $Y^{H}$ is connected and oriented. It holds that $j_{\left(\operatorname{Res}_{H}^{G} Y,\{y\}\right)}^{*}\left(\operatorname{Res}_{H}^{G} \omega\right)=\operatorname{Res}_{H}^{G} 1_{\Omega}-\operatorname{Res}_{H}^{G} \alpha+\left(\operatorname{Res}_{H}^{G} \alpha\right) j_{\left(Y^{H},\{y\}\right)}^{*}\left(\operatorname{Res}_{H}^{G} \omega^{\prime}\right)$ in $\Omega(H)$, and hence $\operatorname{deg}_{H}\left(j_{\left(\operatorname{Res}_{H}^{*} Y,\{y\}\right)}\left(\operatorname{Res}_{H}^{G} \omega\right)\right)=\chi_{H}\left(\operatorname{Res}_{H}^{G} 1_{\Omega}-\operatorname{Res}_{H}^{G} \alpha+\left(\operatorname{Res}_{H}^{G} \alpha\right) j_{\left(Y^{H},\{y\}\right)}^{*}\left(\operatorname{Res}_{H}^{G} \omega^{\prime}\right)\right)$. Since $\chi_{H}\left(\operatorname{Res}_{H}^{G} \alpha\right)=\chi_{H}(\alpha)=0$, it follows from (4.4.3) that $\operatorname{deg}\left(f^{H}\right)=\operatorname{deg}_{H}\left(j_{(Y,\{y\})}^{*}(\omega)\right)$ $=1$. This shows (0.1.3). By $\operatorname{Res}_{H}^{G} \alpha=0$ for $H \in \mathscr{M}(G)$, we also have $\operatorname{Res}_{H}^{G}(\omega)$ $=1_{\operatorname{Res}_{H}^{G} Y}$ in $\omega_{H}^{0}\left(\operatorname{Res}_{H}^{G} Y\right)$. The property $(0.1 .4)$ follows from the last statement in Theorem 4.4. Q.E.D.

The organization of this paper should be in order. $\S 1$ is devoted to basic facts of the Burnside ring and the equivariant cohomology theory. In $\S 2$, we analyze $\mathscr{M}(G)$ in order to find a localizer $\alpha \in \Omega(G)$ of $\omega_{G}^{0}(Y)$. In $\S 3$, the definition of $G$-normal map is given and basic facts of $G$-normal map are discussed. In $\S 4$, an equivariant transversality construction theorem is stated and proved. $\S 5$ is devoted to an equivariant transversality theorem. $\S 6$ is supplement to $\S 3$.

\section{§1. Elements from the Burnside Ring and the Equivariant Cohomology Theory}

Let $G$ be a finite group. Let $\Omega(G)$ be the Burnside ring of $G$ (see, e.g. $[\mathrm{tD} 1, \S 1.2]$ and $[\mathrm{tD} 2$, Chapter I (2.18)]). Thus, $\Omega(G)$ is the Grothendieck 
ring for the category of finite $G$-sets. For a finite $\mathrm{CW}$-complex $A$, let $\chi(A)$ denote the Euler characteristic of $A$. Then we can identify

$$
\Omega(G)=\{[X] \mid X \text { is a finite } G-C W \text {-complex }\}
$$

where $[X]=\left[X^{\prime}\right] \Leftrightarrow \chi\left(X^{H}\right)=\chi\left(X^{\prime H}\right)$ for all $H \leqq G$. (For the proof, compare [tD1, Propositions 1.2.2 and 1.3.5] with [tD2, Chapter IV Proposition 4.6 and Theorem 5.7].)

If $H \leqq G$ then the right coset space $G / H$ has a canonical (left) $G$-action. In particular, $G / G$ consists of a single point and has the trivial action. The unity 1 of $\Omega(G)$ is the equivalence class of $G / G$. For each $H \leqq G$, there is a natural homomorphism $\chi_{H}: \Omega(G) \rightarrow \mathbb{Z}$ such that for $a=[A] \in \Omega(G)(A$ is a finite $G$-CW-complex), $\chi_{H}(a)=\chi\left(A^{H}\right)$. Define $\operatorname{Conj}(G)=\mathscr{S}(G) / G$. Namely, $\operatorname{Conj}(G)$ is the set of all conjugacy classes of subgroups of $G$. For $H \leqq G$, let $(H)$ denote the conjugacy class of $H$. Namely, if we regard $(H)$ as a subset of $\mathscr{S}(G)$ then $(H)=\left\{g H g^{-1} \mid g \in G\right\}$. We define $(H) \leqq(K)(H, K \leqq G)$ to be $H \cong g K^{-1}$ for some $g \in G$. Let $\mathscr{C}(G) \cong \mathscr{S}(G)$ be a complete set of representatives for $\operatorname{Conj}(G)$. If $a \in \Omega(G)$ then integers $\varphi_{H}(a)(H \in \mathscr{C}(G))$ are uniquely determined by

$$
a=\sum_{H \in \mathscr{G}(G)} \varphi_{H}(a)[G / H]
$$

Let $\omega_{G}^{*}(Y, Z)$ be the equivariant cohomology theory: If $(Y, Z)$ is a finite $G$-CW-pair (that is, $Y$ is a finite $G$-CW-complex, and $Z$ is a sub-G-complex of $Y$ ) then

$$
\omega_{G}^{n}(Y, Z)=\underset{V}{\operatorname{colim}}\left[(Y / Z) \wedge V^{\bullet},\left(\mathbb{R}^{n} \oplus V\right)^{\bullet}\right]_{G},
$$

where $V=\mathbb{C}[G]^{m}, V^{\cdot}$ is the one-point compactification of $V$, and $[A, B]_{G}$ stands for the set of all base-pointed $G$-homotopy classes of base-pointed $G$-maps $A \rightarrow B$. Let $\omega_{G}^{*}(Y)=\omega_{G}^{*}\left(Y^{+},+\right)$, where $Y^{+}$is the disjoint union of $Y$ and the reference point + . In particular, if $p t$ is a $G$-set consisting of a single point then

$$
\omega_{G}^{0}(p t)=\underset{V}{\operatorname{colim}}\left[V^{\bullet} \rightarrow V^{\bullet}\right]_{G}
$$

For each $H \leqq G$ there is a natural map $\operatorname{deg}_{H}: \omega_{G}^{0}(p t) \rightarrow \mathbb{Z}$ such that for $\omega=\left(f_{V}\right.$ : $\left.V^{\bullet} \rightarrow V^{\circ}\right)_{V} \in \omega_{G}^{0}(p t), \operatorname{deg}_{H}(\omega)=\operatorname{deg}\left[f_{V}^{H}:\left(V^{H}\right)^{\bullet} \rightarrow\left(V^{H}\right)^{\circ}\right]$. Stabilization of the composition $(Y / Z) \wedge V^{\bullet} \rightarrow\left(\mathbb{R}^{n} \oplus V\right)^{\circ} \rightarrow\left(\mathbb{R}^{n} \oplus V\right)^{\circ}$ defines the right operation of $\omega_{G}^{0}(p t)$ on $\omega_{G}^{*}(Y, Z)$, i.e. $\omega_{G}^{*}(Y, Z) \times \omega_{G}^{0}(p t) \rightarrow \omega_{G}^{*}(Y, Z)$. Furthermore it is known that via this operation, $\omega_{G}^{0}(p t)$ becomes a commutative ring with the unity $1=$ $\left(i d_{V}: V^{\circ} \rightarrow V^{\circ}\right)_{V}$, and $\omega_{G}^{*}(Y, Z)$ becomes an $\omega_{G}^{0}(p t)$-module. In addition, there is a ring isomorphism $\Psi: \Omega(G) \cong \omega_{G}^{0}(p t)$ such that $\chi_{H}=\operatorname{deg}_{H} \circ \Psi$ for all $H \leqq G$ (see [tD1, Theorem 7.6.7] or [tD2, Chapter II Theorem 5.17 and Chapter IV 
Theorem 5.7]). Thus $\omega_{G}^{*}(Y, Z)$ is an $\Omega(G)$-module. If $a \in \Omega(G)$ then let $a_{Y}$ denote the element $p^{*}(\Psi(a)) \in \omega_{G}^{0}(Y)$, where $p: Y \rightarrow p t$ denotes the trivial map. Since the ring $\mathbb{Z}$ is identified with the subring $\mathbb{Z} \cdot 1$ of $\Omega(G)$, for an integer $n$ it is clear what $n_{Y} \in \omega_{G}^{0}(Y)$ stands for. If $\alpha \in \Omega(G)$ then let $\alpha^{-1} \omega_{G}^{0}(Y, Z)$ denote the localization of $\omega_{G}^{0}(Y, Z)$ with respect to the multiplicatively closed set $S=\left\{\alpha, \alpha^{2}, \alpha^{3}, \ldots\right\}$. For $\alpha^{-1} \omega_{G}^{0}(Y, Z)$, we call $\alpha$ a localizer.

Lemma 1.1. Let $(Y, Z)$ be a finite $G-C W$-pair, and $\alpha \in \Omega(G)$. Suppose that (1.1.1) $\operatorname{Res}_{H}^{G} \alpha=0$ in $\Omega(H)$ for all $H \in \operatorname{Iso}(G, Y \backslash Z)$.

Then the inclusion induced map $\alpha^{-1} j_{(Y, Z)}^{*}: \alpha^{-1} \omega_{G}^{*}(Y) \rightarrow \alpha^{-1} \omega_{G}^{*}(Z)$ is an $\alpha^{-1} \Omega(G)$ isomorphism.

Proof. It suffices to show $\alpha^{-1} \omega_{G}^{*}(Y / Z, Z / Z)=\{0\}$. If $G \in$ Iso $(G, Y \backslash Z)$ then the equality obviously holds. Otherwise, Lemma 1.1 follows from [Pe3, Lemma 1.6]. Q.E.D.

Lemma 1.2. Let $(Y, Z)$ and $\alpha$ be as in Lemma 1.1. Let $Z=Z_{1} \coprod \cdots \coprod Z_{l}$ (disjoint union) as sub-G-complexes of $Y$ and let $\omega_{i} \in \omega_{G}^{*}\left(Z_{i}\right)(i=1, \ldots, l)$. Then there exist $\omega \in \omega_{G}^{*}(Y)$ and a natural number s such that $j_{\left(Y, Z_{i}\right)}^{*}(\omega)=\alpha^{s} \omega_{i}$ for all $i=1, \ldots, l$.

Proof. There is a canonical decomposition $\omega_{G}^{*}(Z)=\omega_{G}^{*}\left(Z_{1}\right) \oplus \cdots \oplus \omega_{G}^{*}\left(Z_{l}\right)$. Let $\beta=\left(\omega_{1}, \ldots, \omega_{l}\right) \in \omega_{G}^{*}(Z)$, i.e. $j_{(Z, Z)}^{*}(\beta)=\omega_{i}$. By Lemma 1.1, there exists $\tau \in$ $\alpha^{-1} \omega_{G}^{*}(Y)$ such that $\left(\alpha^{-1} j_{(Y, Z)}^{*}\right)(\tau)=\beta$ in $\alpha^{-1} \omega_{G}^{*}(Z)$. Then it holds that $\left(\alpha^{-1} j_{\left(Y, Z_{i}\right.}^{*}\right)(\tau)$ $=\omega_{i}$ in $\alpha^{-1} \omega_{G}^{*}\left(Z_{i}\right)$. The assertion in Lemma 1.2 follows by definition of localization. Q.E.D.

Local-Global Theorem 1.3. Let $(Y, Z)$ and $\alpha$ be as in Lemma 1.2. For an l-tuple $\left(\gamma_{1}, \ldots, \gamma_{l}\right)$ of $\gamma_{i} \in \Omega(G)$, there exist $\omega \in \omega_{G}^{0}(Y)$ and a natural number $s$ such that $j_{\left(Y, Z_{i}\right)}^{*}(\omega)=\alpha^{s}\left(\gamma_{i}\right)_{Z_{i}}$ in $\omega_{G}^{0}\left(Z_{i}\right)$ for all $i=1, \ldots, l$.

Proof. Apply Lemma 1.2 for $\omega_{i}=\left(\gamma_{i}\right)_{Z_{i}}$. Q.E.D.

When $Y$ is a compact smooth $G$-manifold, $Y$ is regarded as a finite $G$-simplicial complex with respect to some equivariant smooth triangulation.

\section{§ 2. A Way to Find Localizers}

In applications of Theorem 1.3, it is required to find a nice $\alpha \in \Omega(G)$ for localization. This section is devoted to a theory to find such $\alpha$. Original suggestion of the theory appeared in [Mo4]. For a finite group $G$, a family $\mathscr{M}_{G}$ (denoted by $\mathscr{M}(G)$ here) of subgroups of $G$ was introduced in [Mo4, $\S 6]$. Theorem 6.3 of [Mo4] indicated the significance of $\mathscr{M}(G)$ in equivariant surgery theory. Subsequently, E. Laitinen defined another family $\mathscr{L}(G)$ of subgroups of $G$ in an explicit way and proved $\mathscr{M}(G)=\mathscr{S}(G) \backslash \mathscr{L}(G)$ in [LaMo, $\S 1]$. This equality enabled us to handle $\mathscr{M}(G)$ much easier for a general 
group $G$. The current section is a reformulation of related ideas in [LaMo, $\S 1$ ], and the proof of Theorem 2.12 is due to Laitinen's idea proving [LaMo, Theorem 1.3].

Let $S$ be a finite set of primes and

$$
\mathrm{k}_{S}=\prod_{p \in S} p
$$

As convention, $k_{\varnothing}=1$.

Definition 2.1. Define $\mathscr{M}_{S}(G)$ to be a maximal subset of $\mathscr{S}(G)$ (with respect to $\subset)$ such that there exists $\alpha=\alpha\left(\mathscr{M}_{S}(G)\right) \in \Omega(G)$ satisfying

(2.1.1) $\chi_{G}(\alpha) \equiv 1 \bmod k_{S}$, and

(2.1.2) $\operatorname{Res}_{H}^{G}(\alpha)=0$ in $\Omega(H)$ for all $H \in \mathscr{M}_{S}(G)$.

As convention, $\mathscr{M}_{\varnothing}=\mathscr{S}(G)$.

Remark. If $\alpha$ satisfies (2.1.1) and (2.1.2) then $\alpha^{m}$ also satisfies (2.1.1) and (2.1.2) for any natural number $m$. Thus, in general $\alpha=\alpha\left(\mathscr{M}_{S}(G)\right)$ is not uniquely determined by $\mathscr{M}_{S}(G)$.

Lemma 2.2. The following hold:

(2.2.1) $\mathscr{M}_{S}(G)$ is uniquely determined by $G$ and $S$.

(2.2.2) If $S \supseteqq S^{\prime}$ then $\mathscr{M}_{S}(G) \cong \mathscr{M}_{S^{\prime}}(G)$.

(2.2.3) $\mathscr{M}_{S}(G)$ is $G$-invariant (with respect to conjugations).

(2.2.4) $\mathscr{M}_{S}(G)$ is lower closed, i.e. $K \in \mathscr{M}_{S}(G)$ and $H \leqq K \Rightarrow H \in \mathscr{M}_{S}(G)$.

Proof. (2.2.1): We show a sum property: If $\mathscr{M}_{S}(G)_{1}$ and $\mathscr{M}_{S}(G)_{2}$ are maximal subsets of $\mathscr{S}(G)$ as in Definition 2.1 with elements $\alpha_{1}=\alpha\left(\mathscr{M}_{S}(G)_{1}\right)$ and $\alpha_{2}=\alpha\left(\mathscr{M}_{S}(G)_{2}\right)$ satisfying (2.1.1) and (2.1.2), respectively. Then $\alpha=\alpha_{1} \alpha_{2}$ satisfies (2.1.1) and (2.1.2) for the subset $\mathscr{M}_{S}(G)_{1} \cup \mathscr{M}_{G}(G)_{2}$ of $\mathscr{S}(G)$. The assertion of (2.2.1) follows from the sum property.

(2.2.2): The assertion follows from definition.

(2.2.3): The assertion follows from the sum property above.

(2.2.4): The assertion follows from definition. Q.E.D.

Lemma 2.3. It holds that

$$
\mathscr{M}_{S}(G)=\bigcap_{p \in S} \mathscr{M}_{\{p\}}(G) .
$$

Proof. By (2.2.2), $\mathscr{M}_{S}(G) \subseteq \bigcap_{p \in S} \mathscr{M}_{\{p\}}(G)$. We prove the other implication. For each $p \in S$, let $\alpha_{p}=\alpha\left(\mathscr{M}_{\{p\}}(G)\right) \in \Omega(G)$ be as in Definition 2.1. Set

$$
\alpha=(-1)^{|S|+1} \prod_{p \in S}\left(\alpha_{p}-1\right)+1 \text {. }
$$

Then it holds that $\chi_{G}(\alpha) \equiv 1 \bmod k_{S}$ and $\operatorname{Res}_{H}^{G}(\alpha)=0$ for all $H \in \bigcap_{p \in S} \mathscr{M}_{\{p\}}(G)$. Thus $\bigcap_{p \in S} \mathscr{M}_{\{p\}}(G) \subseteq \mathscr{M}_{S}(G)$. Q.E.D. 
Let $S_{G}$ be the set of primes $p$ such that $G$ has a normal subgroup of index $p$, and let

$$
k_{G}=k_{S_{G}}=\prod_{p \in S_{G}} p .
$$

Oliver's Lemma 2.4. Given a finite group $G$, there exists $\gamma \in \Omega(G)$ such that (2.4.1) $\chi_{G}(\gamma)=k_{G}$, and

(2.4.2) $\operatorname{Res}_{H}^{G} \gamma=0$ in $\Omega(H)$ for all $H \neq G$.

Proof. Let $\varphi: \mathscr{S}(G) \rightarrow \mathbb{Z}$ be as in [O, Lemma 8]. Then $\varphi$ is a resolving function in the sense of [O, p. 159]. By [O, Theorem 1], there exists a finite $G$-CW-complex $X$ such that $\chi\left(X^{G}\right)=1+k_{G}$ and $\chi\left(X^{H}\right)=1$ for any $H \neq G$. The element $\gamma=[X]-[G / G]$ satisfies (2.4.1) and (2.4.2). Q.E.D.

Corollary 2.5. Given a prime $p$ not dividing $k_{G}$, it holds that $\mathscr{M}_{\{p\}}(G)=$ $\mathscr{S}(G) \backslash\{G\}$.

Proof. Let $\gamma \in \Omega(G)$ be as in Lemma 2.4. Since $\left(k_{G}, p\right)=1, k_{G}^{m} \equiv 1 \bmod p$ for a suitable natural number $m$. For such $m, \alpha=\gamma^{m}$ satisfies $\chi_{G}(\alpha) \equiv 1 \bmod p$ and $\operatorname{Res}_{H}^{G}(\alpha)=0$ for all $H<G$. Thus one gets $\mathscr{M}_{\{p\}}(G)=\mathscr{S}(G) \backslash\{G\}$. Q.E.D.

Corollary 2.6. If $S \supseteqq S_{G}$ and $S \neq \varnothing$ then $\mathscr{M}_{S}(G)=\mathscr{M}_{S_{G}}(G) \backslash\{G\}$.

Proof. It is clear from definition that $G \notin \mathscr{M}_{S}(G)$ when $S \neq \varnothing$. If $S_{G}=\varnothing$ then by Lemma 2.3 and Corollary 2.5, $\mathscr{M}_{S}(G)=\mathscr{S}(G) \backslash\{G\}=\mathscr{M}_{S_{G}}(G) \backslash\{G\}$. If $\mathrm{S}_{G} \neq \varnothing$ then by Lemma 2.3 and Corollary $2.5, \mathscr{M}_{S}(G)=\mathscr{M}_{S_{G}}(G)=\mathscr{M}_{S_{G}}(G) \backslash\{G\}$. Q.E.D.

Integral Theorem 2.7. For a finite set $S$ of primes such that $S \supseteqq S_{G}$, there exists $\alpha \in \Omega(G)$ satisfying

(2.7.1) $\chi_{G}(\alpha)=1$, and

(2.7.2) $\operatorname{Res}_{H}^{G}(\alpha)=0$ for all $H \in \mathscr{M}_{S}(G)$.

Proof. Let $\alpha=\alpha\left(\mathscr{M}_{S}(G)\right) \in \Omega(G)$ be as in Definition 2.1. Then $\chi_{G}(\alpha)=$ $1+a k_{G}$ for some $a \in \mathbb{Z}$. Let $\gamma \in \Omega(G)$ be as in Lemma 2.4. Then $\alpha^{\prime}=\alpha-a \gamma$ satisfies (2.7.1) and (2.7.2). Q.E.D.

We define

$$
\mathscr{M}(G)=\mathscr{M}_{S_{G}}(G) \backslash\{G\} .
$$

By Theorem 2.7 , this family $\mathscr{M}(G)$ of subgroups of $G$ coincides with the family $\mathscr{M}_{G}$ in $[\mathrm{Mo4}, \S 6]$.

If $p$ is a prime then let $G^{p}$ be the smallest normal subgroup of $G$ such that $G / G^{p}$ is a p-group, i.e. $\left|G / G^{p}\right|=p^{m}$ for some nonnegative integer $m$. For a finite set $S$ of primes define

$$
\mathscr{L}_{S}(G)=\left\{H \in \mathscr{S}(G) \mid H \supseteqq G^{p} \text { for some prime } p \in S\right\} .
$$


As convention, $\mathscr{L}_{\varnothing}(G)=\varnothing$. In addition, define

$$
\mathscr{L}(G)=\mathscr{L}_{S_{G}}(G) \cup\{G\}
$$

Lemma 2.11. The following hold:

(2.11.1) If $S \supseteqq S^{\prime}$ then $\mathscr{L}_{S}(G) \supseteqq \mathscr{L}_{S^{\prime}}(G)$.

(2.11.2) $\mathscr{L}_{S}(G)$ is $G$-invariant (with respect to conjugations).

(2.11.3) $\mathscr{L}_{S}(G)$ is upper closed, i.e. $H \in \mathscr{L}_{S}(G)$ and $H \leqq K \Rightarrow K \in \mathscr{L}_{S}(G)$.

(2.11.4) $\mathscr{L}_{S}(G)=\bigcup_{p \in S} \mathscr{L}_{\{p\}}(G)$.

Proof. Straightforward.

Laitinen's Theorem 2.12. Given a finite group $G$ and a finite set $S$ of primes, it holds that $\mathscr{M}_{S}(G)=\mathscr{S}(G) \backslash \mathscr{L}_{S}(G)$.

Proof. By Lemma 2.3 and (2.11.4), it suffices to prove the case where $S=\{p\}$ for a prime $p$.

$\mathscr{M}_{\{p\}}(G) \cap \mathscr{L}_{\{p\}}(G)=\varnothing$ : Let $H \in \mathscr{L}_{\{p\}}(G)$. Then $H \supseteqq G^{p}$. Let $\alpha \in \Omega(G)$ be as in Definition 2.1. By definition, $\chi_{G}(\alpha) \equiv 1 \bmod p$. By the Smith theory, $\chi_{G}(\alpha) \equiv \chi_{G^{p}}(\alpha) \equiv \chi_{H}(\alpha) \bmod p$. Thus, $\chi_{H}(\alpha)$ cannot be 0 . By definition, $H \notin$ $\mathscr{M}_{\{p\}}(G)$.

$\mathscr{S}(G) \backslash \mathscr{L}_{\{p\}}(G) \subseteq \mathscr{M}_{\{p\}}(G)$ : It suffices to show that for each $H \in \mathscr{K}=\mathscr{S}(G) \backslash$ $\mathscr{L}_{\{p\}}(G)$, there exists $\alpha_{H} \in \Omega(G)$ such that $\chi_{G}\left(\alpha_{H}\right) \equiv 1 \bmod p$ and $\chi_{H}\left(\alpha_{H}\right)=0$. If once this is shown then set

$$
\alpha=\prod_{H \in \mathscr{K}} \alpha_{H}
$$

Since $\mathscr{K}$ is lower closed, it holds that $\operatorname{Res}_{H}^{G}(\alpha)=0$ for each $H \in \mathscr{K}$. Obviously $\chi_{G}(\alpha) \equiv 1 \bmod p . \quad$ Thus $\mathscr{K} \cong \mathscr{M}_{\{p\}}(G)$.

For $K \leqq G$ and a nonnegative integer $q$, let

$$
\mathscr{P}(K, q)=\left\{a \in \Omega(G) \mid \chi_{K}(a) \equiv 0 \bmod q\right\} .
$$

Let $H \in \mathscr{K}$. Required $\alpha_{H}$ is an element $a \in \mathscr{P}(H, 0)$ such that $\chi_{G}(a) \equiv 1 \bmod p$. If there do not exist such elements then $\mathscr{P}(H, 0) \subset \mathscr{P}(G, p)$. By Dress [Dr, Proposition 1], $H^{p}=g G^{p} g^{-1}$ for some $g \in G$. Since $G^{p}$ is a normal subgroup of $G, H^{p}=G^{p}$. Consequently $H \supseteqq G^{p}$ and $H \in \mathscr{L}_{\{p\}}(G)$. This contradicts that we began with $H \in \mathscr{K}=\mathscr{S}(G) \backslash \mathscr{L}_{\{p\}}(G)$. Q.E.D.

Corollary 2.13 ([LaMo, Theorem 1.3]). It holds that $\mathscr{M}(G)=\mathscr{S}(G) \backslash \mathscr{L}(G)$.

Proof. This immediately follows from Theorem 2.12 .

Corollary 2.14. $\mathscr{M}_{S}(G)$ is the empty set if and only if $G$ is a p-group for some prime $p \in S$. 
Proof. If part: Let $G$ be a $p$-group for $p \in S$. If $\alpha \in \Omega(G)$ satisfies $\chi_{G}(\alpha) \equiv$ $1 \bmod k_{S}$ then by the Smith theory, $\chi_{\{1\}}(\alpha) \equiv 1 \bmod p$. Thus it does not hold that $\operatorname{Res}_{\{1\}}^{G}(\alpha)=0$. By definition, $\{1\} \notin \mathscr{M}_{S}(G)$. Since $\mathscr{M}_{S}(G)$ is lower closed, $\mathscr{M}_{S}(G)=\varnothing$.

Only if part: If $\mathscr{M}_{S}(G) \neq \varnothing$ then $\{1\} \in \mathscr{M}_{S}(G)$. By Theorem 2.12, $\{1\} \notin$ $\mathscr{L}_{S}(G)$. Thus, $G=G /\{1\}$ cannot be a $p$-group for any prime $p \in S$. Q.E.D.

Let each of $p$ and $q$ be a prime or 1 . Then define $\mathscr{G}_{p}^{q}$ to be the family of all finite groups $G$ admitting subgroups $P$ and $H$ such that

(2.15) $P \triangleleft H \triangleleft G,|P|=p^{s}$ for some nonnegative integer $s,|G / H|=q^{t}$ for some nonnegative integer $t$, and $H / P$ is a cyclic group.

Further set

$$
\begin{aligned}
\mathscr{G}_{p} & =\bigcup_{q} \mathscr{G}_{p}^{q} \\
\mathscr{G}^{q} & =\bigcup_{p} \mathscr{G}_{p}^{q} \\
\mathscr{G}_{S}^{S^{\prime}} & =\bigcup_{p \in S, q \in S^{\prime}} \mathscr{G}_{p}^{q} \text { (for } S \text { and } S^{\prime} \text { finite sets of primes). }
\end{aligned}
$$

As convention, $\mathscr{G}_{S}^{\varnothing}=\mathscr{G}_{S}^{1}$ and $\mathscr{G}_{\varnothing}^{S^{\prime}}=\mathscr{G}_{1}^{S^{\prime}}$. For a finite group $G$, let $\mathscr{G}_{p}^{q}(G)=$ $\left\{H \leqq G \mid H \in \mathscr{G}_{p}^{q}\right\}$. Similarly we use $\mathscr{G}_{p}(G), \mathscr{G}^{q}(G)$, and $\mathscr{G}_{S}^{S^{\prime}}(G)$.

Proposition 2.17. For finite sets of primes $S$ and $S^{\prime}, G \notin \mathscr{G}_{S}^{S^{\prime}}$ if and only if $\mathscr{G}_{S}^{1}(G) \subseteq \mathscr{M}_{S^{\prime}}(G) \backslash\{G\}$.

Proof. We prove the case $S \neq \varnothing$ and $S^{\prime} \neq \varnothing$. The other cases are left to the reader.

If part: Suppose $G \in \mathscr{G}_{S}^{S^{\prime}}$. By definition, there exists a normal series $P \triangleleft$ $H \triangleleft G$ satisfying (2.15) for some $p \in S$ and $q \in S^{\prime}$. Clearly it holds that $H \in$ $\mathscr{G}_{S}^{1}(G)$. Then by hypothesis, $H \in \mathscr{M}_{S^{\prime}}(G) \backslash\{G\}$. On the other hand, since $G / H$ is a $q$-group, $H \in \mathscr{L}_{\{q\}}(G) \subseteq \mathscr{L}_{S^{\prime}}(G)$. By Theorem $2.12, H \notin \mathscr{M}_{S^{\prime}}(G)$, which is a contradiction.

Only if part: Let $K \in \mathscr{G}_{S}^{1} \backslash\left(\mathscr{M}_{S^{\prime}}(G) \backslash\{G\}\right)$. Then $K \in \mathscr{L}_{S^{\prime}}(G)$ by Theorem 2.12. By definition, $K \supseteqq G^{q}$ for some $q \in S^{\prime}$. Since $K \in \mathscr{G}_{S}^{1}, H=G^{q} \in \mathscr{G}_{S}^{1}$. There exists $P \triangleleft H$ such that $P$ is a $p$-group for some $p \in S$ and $H / P$ is cyclic. Hence $G \in \mathscr{G}_{p}^{q}$. Thus the assumption $G \notin \mathscr{G}_{S}^{S^{\prime}}$ implies $\mathscr{G}_{S}^{1}(G) \subseteq \mathscr{M}_{S^{\prime}}(G) \backslash\{G\}$. Q.E.D.

\section{§3. Definition of $G$-Framed Map}

In the current paper, we assume that each (real) $G$-vector bundle over a finite $G$-CW-complex is equipped with a $G$-invariant Riemannian metric. Let $X$ be a finite $G$-CW-complex, and $\tau$ a $G$-vector bundle over $X$. For $H \leqq G$, $\tau^{H}$ is the $N_{G}(H)$-vector bundle consisting of all elements (in the total space of $\tau$ ) fixed by $H$ over $X^{H}$. Taking the orthogonal complement $\tau_{H}$ to $\tau^{H}$, we 
get the direct sum decomposition as $N_{G}(H)$-vector bundles:

$$
\left.\tau\right|_{X^{H}}=\tau^{H} \oplus \tau_{H} .
$$

As a special case, if $V$ is a real $G$-module, we have the decomposition $V=$ $V^{H} \oplus V_{H}$ as $N_{G}(H)$-modules (with respect to some $G$-invariant inner product on $V$ ). If $\xi$ is a $G$-vector bundle over $Y$ and if $f: X \rightarrow Y$ a $G$-map then it holds $\left(f^{*} \xi\right)_{H}=f^{H^{*}}\left(\xi_{H}\right)$ for all $H \leqq G$. For a real $G$-module $V$ and a $G$-space $X$, let $\varepsilon_{X}(V)$ (or simply $\varepsilon(V)$ if $X$ is clear from context) be the product $G$-vector bundle $X \times V$ (with base space $X$ and fiber $V$ ). We note that $\varepsilon_{X}(V)^{H}=$ $\varepsilon_{X^{H}}\left(V^{H}\right)$ and $\varepsilon_{X}(V)_{H}=\varepsilon_{X^{H}}\left(V_{H}\right)$. Let $b: \tau \rightarrow \xi$ be a $G$-vector bundle map of $G$-vector bundles $\tau$ and $\xi$ over $X$. If $H \leqq G$ then by Schur's lemma, $\left.b\right|_{X^{H}}$ : $\left.\left.\tau\right|_{X^{H}} \rightarrow \xi\right|_{X^{H}}$ is decomposed to $\left.b\right|_{X^{H}}=b^{H} \oplus b_{H}$ as $N_{G}(H)$-vector bundle maps, where $b^{H}: \tau^{H} \rightarrow \xi^{H}$ and $b_{H}: \tau_{H} \rightarrow \xi_{H}$. If $\tau_{i}$ and $\xi_{i}(i=1,2)$ are $G$-vector bundles and $b: \tau_{1} \oplus \tau_{2} \rightarrow \xi_{1} \oplus \xi_{2}$ is a $G$-vector bundle map then we write $b$ in the form

$$
\left(\begin{array}{ll}
b_{11} & b_{12} \\
b_{21} & b_{22}
\end{array}\right) \quad \text { where } \quad b_{i j}=\left.b\right|_{\tau_{i}}: \tau_{i} \rightarrow \xi_{j} .
$$

Let $\tau, \xi$ and $\eta$ are $G$-vector bundles over $X$. If $b: \tau \oplus \eta \rightarrow \xi \oplus \eta$ is a $G$-vector bundle map then $b$ is usually called a stable G-vector bundle map from $\tau$ to $\xi$ and one has usually abused the notation $b: \tau \rightarrow \xi$ if the context is clear.

A $G$-vector bundle map $b: \tau \rightarrow \xi$ is called a G-vector bundle isomorphism if $b$ covers $i d_{X}$ (the identity map on $X$ ).

Definition 3.1 ( $\eta$-Normal Condition). Let $b: \tau \oplus \eta \rightarrow \xi \oplus \eta$ be a $G$-vector bundle isomorphism. For a family $\mathscr{H}$ of subgroups of $G$, we say that $b$ satisfies the $(\eta, \mathscr{H})$-quasinormal condition (resp. $(\eta, \mathscr{H})$-normal condition) if for every $H \in \mathscr{H}, b_{H}: \tau_{H} \oplus \eta_{H} \rightarrow \xi_{H} \oplus \eta_{H}$ has the form

$$
\left(\begin{array}{cc}
c_{H} & d_{H} \\
0 & i d_{\eta_{H}}
\end{array}\right)\left(\operatorname{resp} .\left(\begin{array}{cc}
c_{H} & 0 \\
0 & i d_{\eta_{H}}
\end{array}\right)\right),
$$

where $c_{H}: \tau_{H} \rightarrow \xi_{H}$ and $d_{H}: \tau_{H} \rightarrow \eta_{H}$. In the case $\mathscr{H}=\mathscr{S}(G)$, the $(\eta, \mathscr{H})$-quasinormal condition (resp. $(\eta, \mathscr{H})$-normal condition) is simply called the $\eta$-quasinormal condition (resp. $\eta$-normal condition). In the current paper, if both $b$ and $b^{\prime}$ satisfy the $\eta$-quasinormal condition then a G-regular homotopy $b_{t}: b \sim b^{\prime}$ should be understood that at each level $t, b_{t}$ satisfies the $\eta$-quasinormal condition.

The next lemma easily follows from the definition above.

Lemma 3.2. Let $b: \tau \oplus \eta \rightarrow \xi \oplus \eta$ be a G-vector bundle isomorphism satisfying the $(\eta, \mathscr{H})$-quasinormal condition as in (3.1.1). Then the following hold:

(3.2.1) If $H, \mathrm{~K} \in \mathscr{H}$ and $H \leqq K$ then $\left(c_{K}\right)_{H}=\left.c_{H}\right|_{X^{K}}$. 
(3.2.2) There is a canonical $N_{H}(G)$-regular homotopy

$$
h_{H, t}=\left(\begin{array}{cc}
c_{H} & (1-t) d_{H} \\
0 & i d_{\eta H}
\end{array}\right): b_{H} \sim c_{H} \oplus i d_{\eta_{H}}(t \in[0,1]) .
$$

For a smooth $G$-manifold $X, T(X)$ denotes the tangent bundle over $X$ with canonical $G$-action. If $K \leqq G$ and if $A$ is a sub- $K$-manifold of $X$ then let $v(A, X)$ denote the normal bundle of $A$ in $X$ with canonical $K$-action. It holds that $T(X)_{H}=v\left(X^{H}, X\right)$. If $X$ is an oriented manifold then $-X$ is a copy of $X$ with opposite orientaton to $X$.

Definition 3.3 (G-normal map). Let $\mathscr{H}$ be a family of subgroups of $G$. An $\mathscr{H}$-framed $G$-map is a pair $(f, b)$ of a $G$-map $f:(X, \partial X) \rightarrow(Y, \partial Y)$ of compact, oriented, smooth $G$-manifolds $X$ and $Y$ (possibly with $\partial Y=\varnothing$ ), and a $G$ vector bundle isomorphism $b$ : $T(X) \oplus \eta \rightarrow f^{*} \xi \oplus \eta$ (where $\eta$ is a $G$-vector bundle over $X$, and $\xi$ is a $G$-vector bundle over $Y$ ) such that $b$ satisfies the $(\eta, \mathscr{H})$-normal condition. If $\mathscr{H}=\mathscr{S}(G)$ then an $\mathscr{H}$-framed $G$-map is called simply a G-normal map.

The notion of $\mathscr{H}$-framed $G$-map is introduced because it fits $G$-surgery on $X$ of isotropy types $(H)(H \in \mathscr{H})$.

Definition 3.4 (G-Normal Cobordism). A G-normal cobordism

$$
\left(F: W \rightarrow(I \times Y), B: T(W) \oplus \mathrm{H} \rightarrow \varepsilon_{W}(\mathbb{R}) \oplus\left(\pi_{Y} \circ F\right)^{*} \xi \oplus \mathrm{H}\right)
$$

(where $I=[0,1]$ and $\pi_{Y}: I \times Y \rightarrow Y$ is the canonical projection) between two G-normal maps

$$
\begin{aligned}
& \left(f:(X, \partial X) \rightarrow(Y, \partial Y), b: T(X) \oplus \eta \rightarrow f^{*} \xi \oplus \eta\right) \text { and } \\
& \left(f^{\prime}:\left(X^{\prime}, \partial X^{\prime}\right) \rightarrow(Y, \partial Y), b^{\prime}: T\left(X^{\prime}\right) \oplus \eta^{\prime} \rightarrow f^{\prime *} \xi \oplus \eta^{\prime}\right)
\end{aligned}
$$

is defined in a usual way. Namely, $W$ is a compact, oriented, smooth $G$ manifold such that $\partial W=(-X) \cup\left(\partial_{-} W\right) \cup X^{\prime},(-X) \cap\left(\partial_{-} W\right)=\partial(-X), X^{\prime} \cap\left(\partial_{-} W\right)$ $=\partial X^{\prime}, X \cap X^{\prime}=\varnothing ; F:\left(W,-X, X^{\prime}, \partial_{-} W\right) \rightarrow(I \times Y,\{0\} \times Y,\{1\} \times Y, I \times \partial Y)$ is a $G$-map such that $\left.F\right|_{-X}=f$ and $\left.F\right|_{X^{\prime}}=f^{\prime} ; \mathrm{H}$ is a $G$-vector bundle over $W$ such that $\left.\mathrm{H}\right|_{-X}=\eta$ and $\left.\mathrm{H}\right|_{X^{\prime}}=\eta^{\prime}$; and $B$ is a $G$-vector bundle isomorphism satisfying the $\mathrm{H}$-normal condition and

$$
\begin{aligned}
& \left.B\right|_{-X}=i d_{\varepsilon(\mathbb{R})} \oplus b: \varepsilon_{-X}(\mathbb{R}) \oplus T(-X) \oplus \eta \rightarrow \varepsilon_{-X}(\mathbb{R}) \oplus f^{*} \xi \oplus \eta \quad \text { and } \\
& \left.B\right|_{X^{\prime}}=i d_{\varepsilon(\mathbb{R})} \oplus b^{\prime}: \varepsilon_{X^{\prime}}(\mathbb{R}) \oplus T(-X) \oplus \eta^{\prime} \rightarrow \varepsilon_{X^{\prime}}(\mathbb{R}) \oplus f^{\prime *} \oplus \oplus \eta^{\prime}
\end{aligned}
$$

In the following two theorems, let $\tau$, $\xi$ and $\eta$ be $G$-vector bundles over a finite $G$-CW-complex $X$.

Deformation Theorem 3.5. Let $b: \tau \oplus \eta \rightarrow \xi \oplus \eta$ be a G-vector bundle iso- 
morphism satisfying the $\eta$-quasinormal condition. Let $A$ be a sub-G-complex of $X$. Suppose $\left.b\right|_{A}$ satisfies the $\left.\eta\right|_{A}$-normal condition. Then there exists a $G$ regular homotopy (relative to $A$ ) $b_{t}: b \sim b^{\prime}, t \in[0,1]$, (i.e. $b_{0}=b$ and $b_{1}=b^{\prime}$ ) such that $b^{\prime}$ satisfies the $\eta$-normal condition.

This will be used to construct $G$-normal maps in $\S 4$. The next theorem is not required in the current paper, but it is helpful for our understanding the notion of $G$-normal map.

Lück-Madsen's Theorem 3.6. Let $b: \tau \oplus \eta \rightarrow \xi \oplus \eta$ be a G-vector bundle isomorphism satisfying the $\eta$-quasinormal condition. Then there exists a G-vector bundle isomorphism $\hat{b}: \tau \oplus \varepsilon_{X}\left(\mathbb{R}^{m}\right) \rightarrow \xi \oplus \varepsilon_{X}\left(\mathbb{R}^{m}\right)$ (being stably G-regularly homotopic to $b$ ) for some natural number $m$.

Proofs of Theorems 3.5 and 3.6 are deferred until $\S 6$.

\section{§4. Equivariant Transversality Construction of G-Normal Maps}

Original ideas of the equivariant transversality construction of $G$-normal maps were provided by $\mathrm{T}$. Petrie [Pe1-3]. Some improvements were added by [BMo1-2], [LaMo], [LaMoPa], [Mo1-3], and [MoU].

If $X$ is a compact, oriented, smooth $G$-manifold then $-X$ means a copy of $X$ with the opposite orientation to $X$, and let $+X=X$ (with the same orientation as $X$ ). If $a \in \Omega(G)$ and $\Phi(a)=\left(\varphi_{H}^{ \pm}(a)\right)_{H \in \mathscr{C}(G)}$ is a $2|\mathscr{C}(G)|$-tuple of nonnegative integers such that

$$
a=\sum_{H \in \mathscr{C}(G)}\left(\varphi_{H}^{+}(a)-\varphi_{H}^{-}(a)\right)[G / H],
$$

then define

$$
\begin{gathered}
\Phi(a) X=\Phi_{+}(a) X \coprod \Phi_{-}(a) X, \text { and } \\
\Phi_{ \pm}(a) X=\coprod_{H \in \mathscr{C}(G)} \coprod_{j=1}^{\varphi_{H}^{ \pm}(a)}( \pm X) \times G / H .
\end{gathered}
$$

Define $p_{\Phi(a) X, X}: \Phi(a) X \rightarrow X$ by $p_{\Phi(a) X, X}(x, g H)=x$ for all $x \in \pm X$ and $g H \in G / H$.

In the current section, let $Y$ be a compact, connected, oriented, smooth $G$-manifold possibly with boundary $\partial Y$. If $V=\mathbb{C} \oplus V^{\prime}$ as complex $G$-modules then define ${ }_{ \pm} l_{V}: V \rightarrow V$ to be the maps

$$
\begin{gathered}
V=\mathbb{C} \oplus V^{\prime}=\mathbb{R} \oplus \sqrt{-1} \mathbb{R} \oplus V^{\prime} \rightarrow \mathbb{R} \oplus \sqrt{-1} \mathbb{R} \oplus V^{\prime}=\mathbb{C} \oplus V^{\prime}=V \\
(s, t, v) \mapsto( \pm s, t, v) .
\end{gathered}
$$

This definition of ${ }_{ \pm} l_{V}$ depends on the decomposition $V=\mathbb{C} \oplus V^{\prime}$, but the equivariant regular homotopy class of ${ }_{ \pm} l_{V}$ is unique. Define ${ }_{ \pm} l_{V, Y}: \varepsilon_{Y}(V) \rightarrow$ $\varepsilon_{Y}(V)$ to be $\operatorname{id}_{Y} \times{ }_{ \pm} l_{V}$. Furthermore, if $f: X \rightarrow Y$ is a $G$-map then define ${ }_{ \pm} l_{V, f}$ : 
$\varepsilon_{X}(V) \rightarrow \varepsilon_{Y}(V)$ to be $f \times{ }_{{ }} l_{V}$. If $Z$ is a sub- $G$-simplicial complex of $Y$ (with respect to some equivariant smooth triangulation) then let $\mathrm{RN}(Z)$ be a closed $G$-regular neighborhood (being a sub-G-manifold of $Y$ ) of $Z$ in $Y$. If $Z$ is a sub-K-simplicial complex of $Y$ such that

$$
g Z \cap Z \neq \varnothing \quad(g \in G) \Rightarrow g \in K
$$

then $G Z(\subseteq Y)$ is canonically $G$-homeomorphic to $G \times_{K} Z$. In such a case we write $G Z=G \times_{K} Z$.

Equivariant Transversality Construction Theorem 4.4. Let $Y$ be a compact, connected, oriented, smooth $G$-manifold. Let $Z_{1}, \ldots, Z_{l}$ be sub- $K_{i}$-simplicial complexes of $Y$ such that each $\left(Z_{i}, K_{i}\right)$ satisfies (4.3) and that $G Z_{1}, \ldots, G Z_{l}$ are mutually disjoint. Let $\gamma_{i} \in \Omega\left(K_{i}\right)(i=1, \ldots, l)$ and let $\omega$ be an element in $\omega_{G}^{0}(Y)$ satisfying $j_{\left(\operatorname{Res}_{K_{i}}^{G} Y, Z_{l}\right)}^{*}\left(\operatorname{Res}_{K_{\imath}}^{G} \omega\right)=\left(\gamma_{i}\right)_{Z_{\imath}}$ in $\omega_{K_{l}}^{0}\left(Z_{i}\right)(i=1, \ldots, l)$. Further let $\Phi\left(\gamma_{i}\right)=$ $\left(\varphi_{\bar{H}}^{ \pm}\left(\gamma_{i}\right)\right)_{H \in \mathscr{C}\left(K_{i}\right)}$ be $2\left|\mathscr{C}\left(K_{i}\right)\right|$-tuples of nonnegative integers satisfying (4.1) for $G$ and a replaced by $K_{i}$ and $\gamma_{i}$. Let $\mathrm{RN}\left(Z_{i}\right)$ be $K_{i}$-regular neighborhoods of $Z_{i}$ in $Y$ such that $G \mathrm{RN}\left(Z_{i}\right)=G \times_{K_{i}} \mathrm{RN}\left(Z_{i}\right)$ are $G$-regular neighborhoods of $G Z_{i}=$ $G \times_{K_{1}} Z_{i}$ in $Y$ and that $G \mathrm{RN}\left(Z_{1}\right), \ldots, G \mathrm{RN}\left(Z_{l}\right)$ are mutually disjoint. Then there exists a G-normal map $(f, b)$ (where $f:(X, \partial X) \rightarrow(Y, \partial Y), b: T(X) \oplus \varepsilon_{X}(V) \rightarrow$ $f^{*} T(Y) \oplus \varepsilon_{X}(V), V=\mathbb{C}[G]^{m}$ for some natural number $\left.m\right)$ satisfying the following (4.4.1)-(4.4.3):

(4.4.1) Let $U_{i}=f^{-1}\left(\mathrm{RN}\left(Z_{i}\right)\right)(i=1, \ldots, l)$ then $U_{i} \quad\left(=U_{i,+} \coprod U_{i,-}\right)$ is $K_{i^{-}}$ diffeomorphic to $\Phi\left(\gamma_{i}\right) \operatorname{RN}\left(Z_{i}\right)\left(=\Phi_{+}\left(\gamma_{i}\right) \operatorname{RN}\left(Z_{i}\right) \coprod \Phi_{-}\left(\gamma_{i}\right) \operatorname{RN}\left(Z_{i}\right)\right)$ :

$$
U_{i, \pm} \cong \Phi_{ \pm}\left(\gamma_{i}\right) \mathrm{RN}\left(Z_{i}\right)=\coprod_{H \in \mathscr{C}\left(K_{\imath}\right)} \coprod_{j=1}^{\varphi_{H}^{ \pm}\left(\gamma_{1}\right)}\left( \pm \mathrm{RN}\left(Z_{i}\right)\right) \times K_{i} / H
$$

In addition, $\left.f\right|_{U_{i}}: U_{i} \rightarrow \mathrm{RN}\left(Z_{i}\right)$ coincides with $p_{\Phi\left(\gamma_{i}\right) \mathrm{RN}\left(Z_{i}\right), \mathrm{RN}\left(Z_{i}\right)}$.

(4.4.2) Let $l_{i}: \varepsilon_{U_{1}}(V) \rightarrow \varepsilon_{\mathrm{RN}\left(Z_{1}\right)}(V)$ be the $K_{i}$-maps covering $\left.f\right|_{U_{\mathfrak{\imath}}}$ such that

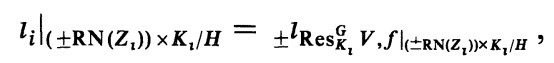

and let $\tilde{f}: f^{*} T(Y) \rightarrow T(Y)$ be the canonical map covering $f$ then $\left.\left(\tilde{f} \oplus l_{V, f}\right)\right|_{U_{\imath}}$ 。 $\left.b\right|_{U_{\imath}}=d f_{U_{\imath}} \oplus \imath_{i}$.

(4.4.3) Let $Y^{H}=\coprod_{c} Y_{c}^{H} \quad$ (connected components), $X_{c}^{H}=X^{H} \cap f^{-1}\left(Y_{c}^{H}\right)$, and $f_{c}^{H}=\left.f\right|_{X_{c}^{H}}: X_{c}^{H} \rightarrow Y_{c}^{H}$ for $H \leqq G$. If $Y_{c}^{H}$ is oriented then $\operatorname{deg}\left(f_{c}^{H}\right)=$ $\operatorname{deg}_{H}\left(j_{\left(\operatorname{Res}_{H}^{G} Y,\{y\}\right)}^{*}\left(\operatorname{Res}_{H}^{G} \omega\right)\right)$ for arbitrary $y \in Y_{c}^{H}$.

Moreover for every $H \leqq G$ such that $\operatorname{Res}_{H}^{G}(\omega)=1_{\operatorname{Res}_{H}^{G} Y}$ in $\omega_{H}^{0}\left(\operatorname{Res}_{H}^{G} Y\right)$, there exists an H-normal cobordism $\left({ }_{H} F,{ }_{H} B\right):(f, b) \sim\left(i d_{\operatorname{Res}_{H}^{G} Y}, i d_{T\left(\operatorname{Res}_{H}^{G} Y\right)} \oplus i d_{\varepsilon\left(\operatorname{Res}_{H}^{G} V\right)}\right)$ for sufficiently large $V$.

The rest of this section is devoted to proving Theorem 4.4.

The outline of our proof is as follows. The element $\omega$ in Theorem 4.4 provides a $G$-map $\alpha: Y \times V^{\cdot} \rightarrow Y \times V^{*}\left(V=\mathbb{C}[G]^{m}\right.$ for some $\left.m\right)$ covering $i d_{Y}$. 
T. Petrie proved that $\alpha$ is equivariantly approximated by a smooth $G$-map $\beta: Y \times V^{\circ} \rightarrow Y \times V^{\circ}$ such that $\beta$ is transversal to $Y \times\{0\}$. Required $f: X \rightarrow Y$ in Theorem 4.3 is obtained by setting $X=\beta^{-1}(Y \times\{0\}), f=\left.p_{Y} \circ \beta\right|_{X}: X \rightarrow Y$ where $p_{Y}: Y \times V \rightarrow Y$ is the canonical projection.

The notion of "normal derivative" defined below is a key to this equivariant approximation and also to realizing the $\varepsilon_{X}(V)$-quasinormal condition (cf. Definitions 3.1 and 3.3). This is one of important ingredients found by $T$. Petrie of equivariant surgery theory.

Definition 4.5 ( $V$-Normal Derivative). Let $V$ be a real $G$-module. If $\alpha: Y \times V \rightarrow Y \times V$ is a $G$-map being smooth at $x \in Y \times V$ then the $V$-normal derivative $v d_{x}(\alpha)$ of $\alpha$ at $x$ is defined to be the composition

$$
V_{G_{x}} \stackrel{i n c l}{\longrightarrow} T_{x}(Y \times V)_{G_{x}} \stackrel{(d \alpha)_{x}}{\longrightarrow} T_{\alpha(x)}(Y \times V)_{G_{x}} \stackrel{\text { proj }}{\longrightarrow} V_{G_{x}},
$$

where $G_{x}$ is the isotropy subgroup at $x$, and $(d \alpha)_{x}$ is the differential of $\alpha$ at $x$.

The next lemma was used as a well-known fact in [BMo1-2], [LaMo], [LaMoPa], [Mo1-3], and [MoU].

Lemma 4.6. Let $V$ be a complex G-module such that $V \supseteqq \mathbb{C}[G]$ and $\gamma \in \Omega(G)$. Let $\Phi(\gamma)=\left(\varphi_{H}^{ \pm}(\gamma)\right)$ be a $2|\mathscr{C}(G)|$-tuple of nonnegative integers satisfying (4.1) for $a=\gamma$. Then there exists a base-pointed $G$-map $h: V^{\circ} \rightarrow V^{\circ}$ satisfying the following (4.6.1)-(4.6.4):

(4.6.1) $\operatorname{deg}\left(h^{H}\right)=\chi_{H}(\gamma)$ for all $H \leqq G$.

(4.6.2) $h$ is transversal to $\{0\}$ in $V$.

(4.6.3) $h^{-1}(0)=h^{-1}(0)_{+} \llbracket h^{-1}(0)_{-}$such that $h^{-1}(0)_{ \pm} \cong \coprod_{H \in \mathscr{C}(G)} \coprod_{j=1}^{\varphi_{H}^{+}(\gamma)}( \pm G / H)$ (as G-sets).

(4.6.4) For each $x \in h^{-1}(0)_{ \pm}$, there is a $G_{x}$-disk neighborhood $D_{x}(V)$ of 0 such that $h\left(D_{x}(V)+x\right)=D_{x}(V)$ and $\left.h\right|_{D_{x}(V)+x}$ coincides with the $G_{x}$-diffeomorphism

$$
D_{x}(V)+x \rightarrow D_{x}(V) ; \quad v+x \mapsto{ }_{ \pm} l_{V}(v) .
$$

In particular, the $V$-normal derivative $v d_{x}(h)=i d_{V_{G_{x}}}$.

Proof. Let $V=\mathbb{C}[G] \oplus V^{\prime}, \Lambda_{ \pm}=\left\{(H, j, \pm) \mid H \in \mathscr{C}(G), j=1, \ldots, \varphi_{\bar{H}}^{ \pm}(\gamma)\right\}$, and $\Lambda=\Lambda_{+} \coprod \Lambda_{-}$. Take distinct $\left|\Lambda_{+}\right| G$-orbits $G x_{H, j,+}$ and $\left|\Lambda_{-}\right| G$-orbits $G x_{H, j,-}$ in $V$ such that the isotropy subgroup at $x_{H, j, \pm}$ is $H$. Let $G \times_{H}$ $U(H, j, \pm)\left((H, j, \pm) \in \Lambda_{ \pm}\right)$be disjoint $G$-tubular neighborhoods of the orbits $G x_{H, j, \pm}$ such that $U(H, j, \pm)=x_{H, j, \pm}+B_{\varepsilon}(V)$ as $H$-spaces, where $B_{\varepsilon}(V)$ is an $\varepsilon$-ball in $V$ centered at the origin for small $\varepsilon>0$. Let $h_{H, j, \pm}: G \times_{H} U(H, j, \pm) \rightarrow$ $V^{\cdot}$ be a smooth $G$-map satisfying the following (i)-(iii).

(i) $h_{H, j, \pm}([1, u])={ }_{ \pm} l_{V}\left(u-x_{H, j, \pm}\right)$ for $u \in U(H, j, \pm)$ close to $x_{H, j, \pm}$ (i.e. $0 \leqq$ $\left.\left\|u-x_{H, j, \pm}\right\| \ll \varepsilon\right)$.

(ii) $h_{H, j, \pm}^{-1,}(0)=G x_{H, j, \pm}$ (the $G$-orbit containing the point $x_{H, j, \pm}$ ). 
(iii) $h_{H, j, \pm}\left(\partial\left(G \times_{H} U_{H, j, \pm}\right)\right)=\{\infty\}$.

These $G$-maps $h_{H, j, \pm}$ together are extended to a $G$-map $h: V^{\bullet} \rightarrow V^{\bullet}$ by setting $h(v)=\infty$ for all $v \notin \bigcup_{(H, j, \pm) \in A} G \times_{H} U(H, j, \pm)$. This map $h$ satisfies the required properties. Q.E.D.

Petrie's Lemma 4.7 (cf. [Pe3, Theorem 2.8], [Pe1, Chapter II]). Let $A$ be a sub-G-simplicial complex of $Y$. Let $\alpha: Y \times V^{\cdot} \rightarrow Y \times V^{\cdot}$ be a G-map such that $\alpha(y, \infty)=(y, \infty)$ for all $y \in Y$ and that $\alpha\left(\partial Y \times V^{\circ}\right) \subseteq \partial Y \times V^{\circ}$. Suppose that $\alpha$ is a smooth map in a neighborhood of $(A \times V) \cap \alpha^{-1}(A \times\{0\})$, and that $\left.\alpha\right|_{A \times V^{*}}$ is transversal to $Y \times\{0\}$ in $A \times V^{\cdot}$ in such a way that for each $x \in$ $\alpha^{-1}(A \times\{0\})$, the $V$-normal derivative $v d_{x}(\alpha)$ is the identity. Then $\alpha$ is $G$ homotopic (relatively to a neighborhood of $\left(A \times V^{*}\right) \cup(Y \times\{\infty\})$ to a $G$-map $\beta$ satisfying the following (4.7.1)-(4.7.4):

(4.7.1) $\beta\left(\partial Y \times V^{*}\right) \subseteq \partial Y \times V^{*}$.

(4.7.2) $\beta$ is a smooth map in a neighborhood of $\beta^{-1}(Y \times\{0\})$, and $\beta$ is transversal to $Y \times\{0\}$ in $Y \times V^{\circ}$.

(4.7.3) $\left.\beta\right|_{\partial Y \times V^{*}}: \partial Y \times V^{\bullet} \rightarrow \partial Y \times V^{\circ}$ is transversal to $\partial Y \times\{0\}$ in $\partial Y \times V^{\bullet}$

(4.7.4) At every point in $\beta^{-1}(Y \times\{0\})$, the $V$-normal derivative of $\beta$ is the identity.

Lemma 4.7 is an analogue of [Pe3, Theorem 2.8]. Methods and arguments of the proof of Lemma 4.7 are same as those of [Pe3, Theorem 2.8]. But the proof of [Pe3, Theorem 2.8] is not given in [Pe3] and it is not easy to understand Lemma 4.7 from [Pe1]. Thus, for the interested reader, we give a proof of Lemma 4.7 in $\S 5$.

Proof of Theorem 4.4. In order to show (4.4.3) after the construction of $f$, we now choose and specify points $y_{H, c} \in Y_{c}^{H}$ for all possible $(H, c), H \leqq G$. Let

$$
\gamma_{H, c}=j_{\left(\operatorname{Res}_{K}^{G} Y,\left\{y_{H, c}\right\}\right)}^{*}\left(\operatorname{Res}_{K}^{G} \omega\right) \in \Omega(K), \quad \text { where } K=G_{y_{H, c}} .
$$

Adding these $\left(\left\{y_{H, c}\right\}, \gamma_{H, c}\right)$ to the given family $\left\{\left(Z_{i}, \gamma_{i}\right)\right\}$, we are allowed to assume that

(4.8) $y_{H, c} \in\left(Z_{1} \cup \cdots \cup Z_{l}\right) \cap Y_{c}^{H}$ for all $H \leqq G$ and connected components $Y_{c}^{H}$. This assumption is made for calculation of $\operatorname{deg}\left(f_{c}^{H}\right)$ by a straightforward method. There is another method to calculate $\operatorname{deg}\left(f_{c}^{H}\right)$ without the assumption (4.8), although we do not discuss this method. Thus, the reader may remember that the assumption (4.8) is inessential.

Set $Z=G Z_{1} \coprod \cdots \coprod G Z_{l}$. The given element $\omega \in \omega_{G}^{0}(Y)$ corresponds to a base-pointed $G$-map $\varphi: Y^{+} \wedge V^{\circ} \rightarrow V^{\circ}$, where $V=\mathbb{C}[G]^{m}$ for some natural number $m$. Define a $G$-map $\alpha: Y \times V^{\bullet} \rightarrow Y \times V^{\bullet}$ covering $i d_{Y}$ by $\alpha(y, v)=$ $(y, \varphi(y, v))$ for $(y, v) \in Y \wedge V^{\circ}$. Note that $\alpha(y, \infty)=(y, \infty)$ for all $y \in Y$. 
Now we consider this correspondence in the opposite direction for $Y$ replaced by $\operatorname{RN}\left(Z_{i}\right)(i=1, \ldots, l)$. By Lemma 4.6, each $\gamma_{i} \in \Omega\left(K_{i}\right)(i=1, \ldots, l)$ provides a self $K_{i}$-map $h_{i}: V^{\circ} \rightarrow V^{\bullet}$ having the properties (4.6.1)-(4.6.4). The map $\alpha_{i}=i d_{\mathrm{RN}\left(Z_{i}\right)} \times h_{i}: \mathrm{RN}\left(Z_{i}\right) \times V^{\circ} \rightarrow \mathrm{RN}\left(Z_{i}\right) \times V^{\circ}$ corresponds to $\left(\gamma_{i}\right)_{\mathrm{RN}\left(Z_{i}\right)}$ in $\omega_{K_{i}}^{0}\left(\mathrm{RN}\left(Z_{i}\right)\right)$. Since $\mathrm{RN}\left(Z_{i}\right)$ is $K_{i}$-homotopy equivalent to $Z_{i}$, it holds that $j_{\left(\operatorname{Res}_{K_{i}}^{G} Y, \operatorname{RN}\left(Z_{i}\right)\right)}^{*}\left(\operatorname{Res}_{K_{i}}^{G} \omega\right)=\left(\gamma_{i}\right)_{\operatorname{RN}\left(Z_{i}\right)}$. Thus we may suppose $\left.\alpha\right|_{\operatorname{RN}\left(Z_{i}\right)}=i d_{\operatorname{RN}\left(Z_{i}\right)} \times h_{i}$.

By Lemma 4.7, $\alpha$ is $G$-homotopic (relatively to $\left.(Y \times\{\infty\}) \cup\left(\operatorname{RN}(Z) \times V^{\circ}\right)\right)$ to a $G$-map $\beta$ satisfying (4.7.1)-(4.7.4). More strictly to say, we should apply Lemma 4.7 to the $G$-map $\left.\alpha\right|_{Y^{\prime} \times V^{\circ}}$ (where $Y^{\prime}=Y \backslash \operatorname{Int}(\operatorname{RN}(Z))$ ) relatively to $\left(Y^{\prime} \times\{\infty\}\right) \cup\left(\partial Y^{\prime} \times V^{\circ}\right)$. Thus, it holds that $\beta^{-1}\left(Y^{\prime} \times V^{\circ}\right) \subseteq Y^{\prime} \times V^{\circ}$ and $\beta^{-1}\left(\mathrm{RN}(Z) \times V^{*}\right) \subseteq \mathrm{RN}(Z) \times V^{*}$.

Let $X=\beta^{-1}(Y \times\{0\})$ and $f=\left.p_{Y} \circ \beta\right|_{X}: X \rightarrow Y$, where $p_{Y}: Y \times V \rightarrow Y$ is the canonical projection. Clearly, $X \subset Y \times V$. The first half of (4.4.1) that $f^{-1}\left(\mathrm{RN}\left(Z_{i}\right)\right)$ is the union of the two spaces

$$
U_{i, \pm} \cong \coprod_{H \in \mathscr{C}\left(K_{i}\right)} \coprod_{j=1}^{\varphi_{H}^{ \pm}\left(\gamma_{i}\right)}\left( \pm \mathrm{RN}\left(Z_{i}\right)\right) \times K_{i} / H
$$

follows from (4.6.3). The latter half of (4.4.1) that $\left.f\right|_{U_{i}}=p_{\Phi\left(\gamma_{i}\right) \mathbf{R N}\left(Z_{i}\right), \operatorname{RN}\left(Z_{i}\right)}$ follows from (4.6.4). Recall the assumption (4.8). By (4.6.4), the map $f_{c}^{H}: X_{c}^{H} \rightarrow Y_{c}^{H}$ is transversal to $\left\{y_{H, c}\right\}$. Thus $\operatorname{deg}\left(f_{c}^{H}\right)$ is computable as follows. Suppose $\left\{y_{H, c}\right\} \in Z_{i}$. Then, $\operatorname{deg}\left(f_{c}^{H}\right)$ is the sum of the signs $\varepsilon(x)$ of $f_{c}^{H}$ at points $x \in$ $f_{c}^{H^{-1}}\left(y_{H, c}\right)$. This sign $\varepsilon(x)$ is defined to be 1 (resp. -1) if $f_{c}^{H}$ preserves (resp. reverses) the orientation at $x$. By (4.6.3), the set $f_{c}^{H^{-1}}\left(y_{H, c}\right)$ is the union of $f_{c}^{H^{-1}}\left(y_{H, c}\right)_{ \pm}$which are identified with

$$
\coprod_{H^{\prime} \in \mathscr{C}\left(K_{i}\right)} \coprod_{j=1}^{\varphi_{H}^{ \pm},\left(\gamma_{i}\right)}\left( \pm K_{i} / H^{\prime}\right)^{H}
$$

By (4.6.4),

$$
\begin{aligned}
& \varepsilon(x)=1 \text { on } \coprod_{H^{\prime} \in \mathscr{C}\left(K_{i}\right)} \coprod_{j=1}^{\varphi_{H}^{+},\left(\gamma_{1}\right)}\left(K_{i} / H^{\prime}\right)^{H}, \quad \text { and } \\
& \varepsilon(x)=-1 \text { on } \coprod_{H^{\prime} \in \mathscr{C}\left(K_{\imath}\right)} \coprod_{j=1}^{\varphi_{H}^{-},\left(\gamma_{1}\right)}\left(-K_{i} / H^{\prime}\right)^{H} .
\end{aligned}
$$

It follows that

$$
\begin{aligned}
\operatorname{deg}\left(f_{c}^{H}\right) & =\left|\coprod_{H^{\prime} \in \mathscr{C}\left(K_{i}\right)} \coprod_{j=1}^{\varphi_{H}^{+},\left(\gamma_{2}\right)}\left(K_{i} / H^{\prime}\right)^{H}\right|-\left|\coprod_{H^{\prime} \in \mathscr{C}\left(K_{\imath}\right)} \coprod_{j=1}^{\varphi_{H}^{-},\left(\gamma_{v}\right)}\left(-K_{i} / H^{\prime}\right)^{H}\right| \\
& =\chi_{H}\left(\gamma_{i}\right)=\operatorname{deg}_{H}\left(j_{\left(\operatorname{Res}_{H}^{G} \boldsymbol{Y},\left\{y_{H, c}\right\}\right)}^{*}\left(\operatorname{Res}_{H}^{G} \omega\right)\right) .
\end{aligned}
$$

This shows the property (4.4.3).

Next we see the existence of $b$ stated in Theorem 4.4. Let $v_{X}=$ $v(X, Y \times V)$ and $v_{Y}=v(Y, Y \times V)$. Let $p_{V}: Y \times V \rightarrow V$ be the canonical 
projection, and $\tau: v_{X} \rightarrow v_{Y}$ a standard $G$-vector bundle map covering $f: X \rightarrow Y$, namely

$$
\tau:\left.\left.v_{X} \hookrightarrow T(Y \times V)\right|_{X} \stackrel{d \beta}{\longrightarrow} T(Y \times V)\right|_{Y} \stackrel{\text { proj }}{\longrightarrow} v_{Y} .
$$

Define $\tau^{\prime}: v_{X} \rightarrow f^{*} v_{Y}$ by $\tau^{\prime}(w)=(\pi(w), \tau(w))$ for $w \in v_{X}$, where $\pi: v_{X} \rightarrow X$ is the projection. By the equivariant covering homotopy property, there is a metricpreserving $G$-vector bundle isomorphism $\gamma: \alpha^{*}\left(p_{Y}^{*}(T(Y))\right) \cong \beta^{*}\left(p_{Y}^{*}(T(Y))\right)$. Then it holds that

$$
\begin{aligned}
T(X) \oplus \varepsilon_{X}(V) & =T(X) \oplus f^{*} \varepsilon_{Y}(V) \\
& =T(X) \oplus f^{*} v_{Y} \\
& \simeq T(X) \oplus v_{X} \quad\left(\text { via id } \oplus \tau^{\prime-1}\right) \\
& =\left.T(Y \times V)\right|_{X} \\
& =\left.\left(p_{Y}^{*}(T(Y)) \oplus p_{V}^{*}(T(V))\right)\right|_{X} \\
& =\left.\left.p_{Y}^{*}(T(Y))\right|_{X} \oplus p_{V}^{*}\left(\varepsilon_{V}(V)\right)\right|_{X} \\
& =\left.p_{Y}^{*}(T(Y))\right|_{X} \oplus \varepsilon_{X}(V) \\
& =\left.\alpha^{*}\left(p_{Y}^{*}(T(Y))\right)\right|_{X} \oplus \varepsilon_{X}(V) \\
& \left.\cong \beta^{*}\left(p_{Y}^{*}(T(Y))\right)\right|_{X} \oplus \varepsilon_{X}(V) \\
& =\left.\left.\beta^{*}\left(p_{Y}^{*}(T(Y))\right)\right|_{X} \oplus \beta^{*}\left(p_{V}^{*}\left(\varepsilon_{V}(V)\right)\right)\right|_{X} \\
& =\left.\left.\beta^{*}\left(p_{Y}^{*}(T(Y))\right)\right|_{X} \oplus \beta^{*}\left(p_{V}^{*}(T(V))\right)\right|_{X} \\
& =\left.\beta^{*}\left(p_{Y}^{*}(T(Y)) \oplus p_{V}^{*}(T(V))\right)\right|_{X} \\
& =\left.\beta^{*}(T(Y \times V))\right|_{X} \\
& =f^{*}\left(\left.T(Y \times V)\right|_{Y}\right) \\
& =f^{*}\left(T(Y) \oplus v_{Y}\right) \\
& =f^{*}(T(Y)) \oplus f^{*}\left(v_{Y}\right) \\
& =f^{*}(T(Y)) \oplus f^{*}\left(\varepsilon_{Y}(V)\right) \\
& =f^{*}(T(Y)) \oplus \varepsilon_{X}(V),
\end{aligned}
$$

where $=($ resp. $\cong, \simeq)$ should be read "isomorphic (via a canonical isomorphism) to" (resp. "isomorphic (via a metric-preserving isomorphism) to", "isomorphic to"). Let $b: T(X) \oplus \varepsilon_{X}(V) \rightarrow f^{*}(T(Y)) \oplus \varepsilon_{X}(V)$ be the composite of these isomorphisms. By (4.9) defining $b$, the property (4.4.2) follows from (4.6.4). Recall that the $V$-normal derivatives $v d_{x}(\beta)$ 's $(x \in X)$ are the identities. By straightforward observation of the each isomorphism in (4.9), we can check that 
$b$ satisfies the $\varepsilon_{X}(V)$-quasinormal condition. By Theorem 3.5, we can arrange $b$ so that it satisfies the $\varepsilon_{X}(V)$-normal condition.

We leave the proof of the existence of $H$-normal cobordism $\left({ }_{H} F,{ }_{H} B\right)$ to the reader, since the proof is quite similar to the above arguments and there are no additional difficulties. Q.E.D.

\section{§5. An Equivariant Transvarsality Theorem}

Let $\left(X, X_{0}\right)$ (resp. $\left.\left(Y, Y_{0}\right)\right)$ be a compact $G$-CW-pair such that $X_{1}=X \backslash X_{0}$ (resp. $Y_{1}=Y \backslash Y_{0}$ ) is a smooth $G$-Riemannian manifold (i.e. $G$-manifold with $G$ invariant Riemannian metric). For $x \in X_{1}$, a map $f: X \rightarrow Y$ such that $f(x) \in Y_{1}$ is said to be smooth at $x$ if $f$ is smooth in a neighborhood of $x$. For $A \subseteq X_{1}$, a map $f: X \rightarrow Y$ such that $f(A) \subseteq Y_{1}$ is said to be smooth on $A$ if $f$ is smooth at every point in $A$. If $f: X \rightarrow Y$ is smooth at $x \in X_{1}\left(f(x) \in Y_{1}\right)$ then $d f_{x}$ : $T_{x}\left(X_{1}\right) \rightarrow T_{f(x)}\left(Y_{1}\right)$ denotes the differential of $f$ at $x$.

Definition 5. 1 (Transversality). Let $L$ be a compact submanifold of $Y_{1}$ (possibly with boundary) with normal bundle $v\left(L, Y_{1}\right)$, and let $A \subseteq X$. A map $f: X \rightarrow Y$ with $f^{-1}(L) \subset X_{1}$ is said to be transversal to $L$ on $A$ if $f$ is smooth on $A \cap f^{-1}(L)$ and proj $\circ d f_{x}: T_{x}\left(X_{1}\right) \rightarrow T_{f(x)}\left(Y_{1}\right) \rightarrow v_{f(x)}\left(L, Y_{1}\right)$ is surjective for all $x \in A \cap f^{-1}(L)$.

Remark 5.2. The transversality is an open property. Namely, if $f$ is transversal to $L$ on $A$ then so is $f$ on a neighborhood of $A$.

Let $V$ and $W$ be real $G$-modules with $G$-invariant inner product, $l: \varepsilon_{X_{1}}(V) \rightarrow$ $T\left(X_{1}\right)$ a metric preserving, $G$-vector bundle monomorphism (covering $i d_{X_{1}}$ ), and $\pi: T\left(Y_{1}\right) \rightarrow \varepsilon_{Y_{1}}(W)$ a $G$-vector bundle epimorphism (covering $i d_{Y_{1}}$ ).

Definition $5.3\left((l, \pi)\right.$-Normal Derivative). Let $x \in X_{1}$, and let $f: X \rightarrow Y$ be a $G$-map smooth at $x\left(f(x) \in Y_{1}\right)$. Then the $(l, \pi)$-normal derivative $v d_{x}(f): \mathbb{V}_{G_{x}} \rightarrow$ $W_{G_{x}}$ of $f$ at $x$ is defined to be the composition

$$
V_{K} \stackrel{l_{x K}}{\longrightarrow} T_{x}\left(X_{1}\right)_{K} \stackrel{d f_{x K}}{\longrightarrow} T_{f(x)}\left(Y_{1}\right)_{K} \stackrel{\pi_{f(x) K}}{\longrightarrow} W_{K} \quad\left(K=G_{x}\right) .
$$

If $X_{1}=A \times V$ and $Y_{1}=B \times W$ (as G-Riemannian manifolds) and if

$$
\begin{aligned}
& l: \varepsilon_{A \times V}(V) \rightarrow T(A \times V)=p_{A}^{*} T(A) \oplus p_{V}^{*} \varepsilon_{V}(V), \quad \text { and } \\
& \pi: T(B \times W)=p_{B}^{*} T(B) \oplus p_{W}^{*} \varepsilon_{W}(W) \rightarrow \varepsilon_{B \times W}(W)
\end{aligned}
$$

are the canonical maps (i.e.

$$
\begin{gathered}
l\left((a, v), v^{\prime}\right)=\left(0_{(a, v)},\left((a, v),\left(v, v^{\prime}\right)\right)\right) \quad\left((a, v) \in A \times V, v^{\prime} \in V\right), \quad \text { and } \\
\pi\left(\left(z,\left((b, w),\left(w, w^{\prime}\right)\right)\right)=\left((b, w), w^{\prime}\right) \quad\left((b, w) \in B \times W,\left.z \in\left(p_{B}^{*} T(B)\right)\right|_{(b, w)}, w^{\prime} \in W\right)\right),
\end{gathered}
$$


then the $(l, \pi)$-normal derivative is called the $(V, W)$-normal derivative. Furthermore if $V=W$ then $(V, W)$-normal derivative is simply called the $V$-normal derivative (cf. Definition 4.5).

Let $\eta: V \rightarrow W$ be a $G$-epimorphism (i.e. an epimorphism being a $G$-map).

Definition 5.4. In the situation of Definition 5.1, further suppose that $L$ is a compact smooth $G$-manifold, $\left(Y_{1}, L\right)=(L \times W, L \times\{0\})$ as $G$-Riemannian manifolds, and $\pi: T\left(Y_{1}\right) \rightarrow \varepsilon_{Y_{1}}(W)$ is the canonical map. A $G$-map $f: X \rightarrow Y$ with $f^{-1}(L \times\{0\}) \subset Y_{1}$ is said to be $(l, \pi ; \eta)$-transversal (or simply $\eta$-transversal if the context is clear) to $L \times\{0\}$ on $A$ if $f$ is transversal to $L \times\{0\}$ on $A$ and if the $(l, \pi)$-normal derivative $v d_{x}(f)$ of $f$ coincides with $\eta_{G_{x}}$ at every $x \in A \cap f^{-1}(L \times\{0\})$.

In the following, let $Y$ possess a $G$-invariant distance function $d: Y \times Y \rightarrow$ $\mathbb{R}$.

Equivariant Transversality Theorem 5.5. (In the situation of Definition 5.4.) Let $A$ be a sub-G-CW complex of $X$ such that $A \supseteqq X_{0} \cup \partial X_{1}$. Suppose that $f\left(X_{0}\right) \subseteq Y_{0}$, that $f\left(\partial X_{1}\right) \subseteq \partial Y_{1}(=\partial L \times W)$, and that $f$ is $\eta$-transversal to $L \times\{0\}$ in $Y_{1}$ on $A$. If $\varepsilon>0$ and a sub-G-CW-complex $B \supseteqq A$ then there exists $a$ G-homotopy (relative to $A$ ) $f_{t}: f \sim f^{\prime}\left(f_{0}=f, f_{1}=f^{\prime}\right.$, and at each level $t \in[0,1]$ $f_{t}: X \rightarrow Y$ ) satisfying the following conditions:

(5.5.1) $d\left(f_{t}(x), f(x)\right)<\varepsilon$ for all $t \in[0,1]$ and $x \in X$.

(5.5.2) $f^{\prime}$ is $(l, \pi ; \eta)$-transversal to $L \times\{0\}$ on $B$.

Lemma 5.6. Suppose the situation in Theorem 5.5. Then the following hold:

(5.6.1) $f^{-1}(L \times\{0\}) \subset X_{1}$.

(5.6.2) There exists a compact sub-G-manifold of $Z \subset X_{1}$ such that Int $(Z) \supset$ $f^{-1}(L \times\{0\})$.

Since the proof is easy, we omit it.

Let $K$ be a compact subset of $X_{1}$. $\mathscr{F}(X, Y ; K)$ be the set of all (continuous) maps $h: X \rightarrow Y$ such that $h(K) \subset Y_{1}$ and $h$ is smooth on $K$. The $\mathscr{C}_{W, K}^{1}$ topology on the set $\mathscr{F}(X, Y ; K)$ may be defined as follows. Let $\left\{U_{\alpha}\right\}$ be a finite coordinate covering of $K$ with coordinate maps $h_{\alpha}: U_{\alpha} \rightarrow \mathbb{R}^{n}$ (or $\mathbb{R}_{+} \times$ $\mathbb{R}^{n-1}$, where $\left.\mathbb{R}_{+}=\{x \in \mathbb{R} \mid x \geqq 0\}\right)$, and let $\left\{C_{\alpha}\right\}$ be a compact refinement, of $\left\{U_{\alpha}\right\}$, covering $K$. Let $k \in \mathscr{F}(X, Y ; K)$. For a positive constant $\delta>0$, define a subset $N(k, \delta ; K)$, of $\mathscr{F}(X, Y ; K)$, consisting of all maps $g: X \rightarrow Y$ such that $d(g(x), k(x))<\delta$ for any $x \in X$, and

$$
\left\|\frac{\partial g_{\alpha}}{\partial x_{i}}-\frac{\partial k_{\alpha}}{\partial x_{i}}\right\|<\delta
$$

at all points in $h_{\alpha}\left(C_{\alpha} \cap K\right)$, where $g_{\alpha}=\pi \circ g \circ h_{\alpha}^{-1}$ and $i=1, \ldots, n$. If we take 
the sets $N(k, \delta ; K)(\delta>0)$ as a base of neighborhoods of $k$, the resulting topology is called the $\mathscr{C}_{W, K}^{1}$ topology.

Proof of Theorem 5.5. Set $T=f^{-1}(L \times\{0\})$. By Remark 5.2, (5.6.2), and the equivariant smooth approximation theorem, there exists a closed $G$ invariant subset $A^{\prime}$ of $X$ and a $G$-map $f^{\prime \prime}$ such that $A \subseteq A^{\prime}$, that a neighborhood of $\partial X_{1} \subseteq A^{\prime}$, that $X_{2}=X_{1} \backslash \operatorname{Int}\left(A^{\prime}\right)$ is a compact sub-G-manifold of $X_{1}$ with boundary $X_{2} \cap A^{\prime}$, that $\left.f^{\prime \prime}\right|_{A}=\left.f\right|_{A}$, that $f^{\prime \prime}$ is smooth on $X_{2} \cup T$, that $f^{\prime \prime} \in N(f, \varepsilon / 2 ; \varnothing)$, and that $f^{\prime \prime}$ is $\eta$-transversal to $L \times\{0\}$ on $A^{\prime}$. We may proceed the proof replacing $A$ and $f$ by such $A^{\prime}$ and $f^{\prime \prime}$. Thus we regard $A^{\prime}=A$ and $f^{\prime \prime}=f$. Note that $X_{2}=X_{1} \backslash \operatorname{Int}(A)$ is a finite sub-G-simplicial complex of $X_{1}$. Let $\left\{H_{1}, \ldots, H_{m}\right\}$ be a complete set of representatives for Iso $\left(G, X_{2}\right) / G$ such that $\left(H_{i}\right) \geqq\left(H_{j}\right)$ (subconjugate) $\Rightarrow i \leqq j$. Since $X=A \cup$ $\bigcup_{i=1}^{m} G\left(X_{2}^{H_{i}}\right)$, it suffices to prove Theorem 5.5 in the case where $B=A \cup G\left(X_{2}^{H}\right)$ such that $X_{2}^{K} \subset A$ for all $K \supsetneqq H$. Let $Z=X_{2}^{H}$. Then $Z$ is a free $N / H$-manifold with boundary $Z \cap A$, where $N=N_{G}(H)$.

Note that there exists a positive number $\delta^{\prime}<\varepsilon / 2$ such that if a $G$-map $h: X \rightarrow Y$ with $h \in N\left(f, \delta^{\prime} ; X_{2} \cup T\right)$ then $h$ is $G$-homotopic to $f$ and $h$ is transversal to $L \times\{0\}$ on $A$. In the following, deformation of $f$ should be understood to be done within $\delta^{\prime}$-approximations, and let $\delta=\delta^{\prime} / m$ for a large natural number $m$.

If there exists $f^{\prime}$ as in Theorem 5.5 then for $x \in Z \cap f^{\prime-1}(L \times\{0\})$,

$$
\pi_{f(x)} \circ d f_{x}^{\prime}: T_{x}\left(X^{\prime}\right)=T_{x}(Z) \oplus v_{x}(Z, U) \rightarrow W=W^{H} \oplus W_{H}, \quad\left(U=X_{1} \backslash \operatorname{Int}(A)\right)
$$

must be surjective. Thus we first achieve the surjectivity of $T_{x}(Z) \rightarrow V^{H}$ and next obtain the surjectivity $T_{x}\left(X^{\prime}\right)_{H} \rightarrow W_{H}$. By ordinary transversality theorem, there is a smooth $N$-homotopy $\gamma_{t}:\left.f\right|_{Z} \sim \gamma_{1}$ (at each level $t \in[0,1], \gamma_{t}: Z \rightarrow$ $\left.(L \times W)^{H}\right)$ such that $\gamma_{t} \in N\left(\left.f\right|_{Z}, \delta ; Z\right)$, and $\gamma_{1}$ is transversal to $(L \times\{0\})^{H}$ in $(L \times W)^{H}$ on $Z$. For sufficiently small $\delta$, there exist a compact $N$-submanifold $Z^{\prime} \subset Z$ and an $N$-homotopy $h_{t}:\left.f\right|_{Z} \sim h_{1}$ such that $h_{t} \in N\left(\left.f\right|_{Z}, 2 \delta ; Z\right),\left.h_{t}\right|_{\partial Z}=\left.f\right|_{\partial Z}$ and $h_{1}$ is transversal to $(L \times\{0\})^{H}$ in $(L \times W)^{H}$ on $Z^{\prime}$.

We regard $\varepsilon_{X_{1}}(V) \subseteq T\left(X_{1}\right)$ via $l$. In particular $\varepsilon_{Z}\left(V_{H}\right) \subseteq\left(\left.T\left(X_{1}\right)\right|_{Z}\right)_{H}$. Define $\eta_{h_{t}}: \varepsilon_{Z}\left(V_{H}\right) \rightarrow \varepsilon_{(L \times W)^{H}}\left(W_{H}\right)$ by $\eta_{h_{t}}(x, v)=\left(h_{t}(x), \eta_{H}(v)\right)$ for $x \in Z$ and $v \in V_{H}$. Clearly $\left[\left.\eta_{h_{t}}\right|_{x}: V_{H} \rightarrow W_{H}\right]=\eta_{H}$ and this is a surjective map. Take a smooth $N$-equivariant extension $\lambda_{t}: D_{r} \rightarrow v\left((L \times W)^{H}, L \times W\right)$ of $\eta_{h_{t}}$, where $D_{r}=$ $D_{r}(v(Z, U))$ the closed disk bundle with radius $r>0$ associated to $v(Z, U)$.

Let Exp: $T\left(X_{1}\right) \rightarrow X_{1}$ be the exponential map. Without loss of generality, we may suppose $\operatorname{Exp}\left(D_{r}\right) \subset U$. Let $\operatorname{Exp}^{\prime}: D_{r} \rightarrow U$ be the restriction of $\operatorname{Exp}$. By equivariant tubular neighborhood theorem, if $r>0$ is sufficiently small then Exp' becomes an $N$-embedding and $E=\operatorname{Im}\left(\operatorname{Exp}^{\prime}\right)$ is a closed $N$-tubular neighborhood of $Z$ in $U$. We may suppose that $g \in G$ and $g E \cap E \neq \varnothing \Rightarrow$ 
$g \in N$. Let exp: $v\left((L \times W)^{H}, L \times W\right) \rightarrow L \times W$ be the exponential map. Then we have an $N$-homotopy $k_{t}=\exp \circ \lambda_{t} \circ \operatorname{Exp}^{\prime-1}$ (at each level $t, k_{t}: E \rightarrow L \times W$ ). By construction, $\left.k_{t}\right|_{Z}=h_{t}$, and $k_{1}$ is $\operatorname{Res}_{N}^{G} \eta$-transversal on $Z$. If we take sufficiently small $r>0$ then $\left.k_{t}\right|_{\partial_{+} E} \in N\left(\left.f\right|_{\partial_{+} E}, 3 \delta ; \partial_{+} E\right)$, where $\partial_{+} E=\operatorname{Im}\left(\left.\operatorname{Exp}^{\prime}\right|_{\partial z}\right)$. Thus, if we take $\delta>0$ and $r>0$ so small then there exists a smooth $N$ homotopy $k_{t}^{\prime}:\left.f\right|_{E} \sim k_{1}^{\prime}\left(k_{t}^{\prime}: E \rightarrow L \times W\right)$ such that $d\left(k_{t}^{\prime}(x), f(x)\right)<4 \delta$ for all $x \in E$, that $\left.k_{t}^{\prime}\right|_{\partial_{+} E}=\left.f\right|_{\partial_{+} E}$, and that $k^{\prime}{ }_{1}$ is $\operatorname{Res}_{N}^{G} \eta$-transversal to $L \times\{0\}$ on $Z$. The homotopy $\left.f\right|_{A} \cup k_{t}^{\prime}:\left.\left.f\right|_{A \cup E} \sim f\right|_{A} \cup k^{\prime}{ }_{1}(t \in[0,1])$ is uniquely extensible to a G-homotopy $f^{\prime \prime}{ }_{t}:\left.f\right|_{A \cup G E} \sim f^{\prime \prime}{ }_{1}\left(f^{\prime \prime}{ }_{t}: A \cup G E \rightarrow Y\right.$ at each level $\left.t\right)$. Take a $G$ homotopy $f_{t}: f \sim f_{1}\left(f_{t}: X \rightarrow Y\right)$ such that $d\left(f_{t}(x), f(x)\right)<5 \delta$ and $\left.f_{t}\right|_{A \cup G E}=f^{\prime \prime}{ }_{t}$. Then $f_{t}$ and $f^{\prime}=f_{1}$ are desired ones. Q.E.D.

Proof of Lemma 4.7. Let $Y^{\prime}=Y \cup_{\partial Y}(-Y)$ be the double of $Y$. Then $G^{\prime}=G \times\left\langle\sigma \mid \sigma^{2}=1\right\rangle$ acts on $Y^{\prime}$ in a canonical way. Namely $\left.\sigma\right|_{Y}: Y \rightarrow-Y$ and $\left.\sigma\right|_{-Y}:-Y \rightarrow Y$ are the canonical maps. The $G$-module $V$ can be regarded as a $G^{\prime}$-module where $\sigma$ acts trivially. Let $\alpha^{\prime}: Y^{\prime} \times V^{\circ} \rightarrow Y^{\prime} \times V^{\bullet}$ be the $G^{\prime}$-map canonically obtained from the G-map $\alpha: Y \times V^{\cdot} \rightarrow Y \times V^{*}$. Note that if $\alpha^{\prime}$ is transversal to $Y^{\prime} \times\{0\}$ in $Y^{\prime} \times V^{\circ}$ then $\left.\alpha^{\prime}\right|_{\partial Y}=\left.\alpha\right|_{\partial Y}$ is transversal to $\partial Y \times\{0\}$ in $\partial Y \times V^{\circ}$. Thus apply Theorem 5.5 to the $G^{\prime}$-map $\alpha^{\prime}$ and obtain a $G^{\prime}-$ homotopy (relative to $\left.\left(A^{\prime} \times V^{\bullet}\right) \cup(Y \times\{\infty\})\right) \alpha_{t}^{\prime}: \alpha^{\prime} \sim \beta^{\prime}$ such that $\beta^{\prime}: Y^{\prime} \times V^{\bullet} \rightarrow$ $Y^{\prime} \times V^{\circ}$ is $\left(\imath^{\prime}, \pi^{\prime} ; i d_{V}\right)$-transversal to $Y^{\prime} \times\{0\}$, where $\imath^{\prime}: \varepsilon_{Y^{\prime} \times V}(V) \rightarrow T\left(Y^{\prime} \times V\right)$ and $\pi^{\prime}: T\left(Y^{\prime} \times V\right) \rightarrow \varepsilon_{Y^{\prime} \times V}(V)$ are the canonical maps, and $i d_{V}: V \rightarrow V$ is the identity map on $V$. Then $\beta=\left.\beta^{\prime}\right|_{Y \times V^{*}}: Y \times V^{\bullet} \rightarrow Y \times V^{\bullet}$ is a desired map. Q.E.D.

\section{§6. Supplement}

We begin this section with preparing notation. If $\tau$ is a $G$-vector bundle over $X$ and $x \in X$ then let $\tau_{x}$ be the fiber of $\tau$ at $x$. If $U$ and $V$ be real $G$-modules then $G L_{G}(U, V)$ is the space of all $G$-linear isomorphisms $U \rightarrow V$.

Proof of Theorem 3.5 $\Rightarrow$ Theorem 3.6. By Theorem 3.5, we may suppose that $b$ satisfies the $\eta$-normal condition. Since $X$ is a finite $G$-CW-complex, there exists a real $G$-module $V$ such that $\varepsilon_{X}(V) \supseteqq \eta$. For such $V$, the canonical stabilization of $b$ satisfies the $\varepsilon_{X}(V)$-normal condition. Thus we may suppose $\eta=\varepsilon_{X}(V)$, and in addition $\operatorname{dim} V^{G} \geqq \operatorname{dim} X+1$. A $G$-vector bundle isomorphism $\hat{b}$ as in the conclusion of Theorem 3.6 is obtained by the argument in the proof of [LüMa, Appendix Proposition (A2)] (for $\mathbb{R}^{m}=V^{G}$ ). We give here an outline of the argument, and the interested reader can find the details in [LüMa1, pp. 524-525]. Let $\mathbb{R}^{m}=V^{G}$. The usual cell-by-cell construction of $\hat{b}$ is applicable. Thus suppose that $\hat{b}_{A}$ was already constructed over a sub-G-complex $A$ of $X$. Let $B=A \cup\left(G / H \times D^{i}\right)$ be a sub-G-complex of $X$. In order to obtain $\hat{b}_{B}$, it suffices to extend $N$-equivariantly $\operatorname{Res}_{N}^{G} \hat{b}_{A}\left(N=N_{G}(H)\right)$ over $N$-space $Y=A \cup\left(N / H \times D^{i}\right)$. Take a point $x \in H / H \times \operatorname{Int}\left(D^{i}\right)$. The ob- 
struction to extending $\operatorname{Res}_{N}^{G} \hat{b}_{A}$ to $\hat{b}_{Y}$ over $Y$ such that $\hat{b}_{Y} \oplus i d_{\varepsilon_{Y}\left(V_{G}^{H}\right)}=\left.b^{H}\right|_{Y}$ lies in

$$
\pi_{i}\left(\varphi: G L_{H}\left(\tau_{x}^{H} \oplus V^{G}, \xi_{x}^{H} \oplus V^{G}\right) \rightarrow G L_{H}\left(\tau_{x}^{H} \oplus V^{H}, \xi_{x}^{H} \oplus V^{H}\right)\right) \quad\left(i \leqq \operatorname{dim} X^{H}\right),
$$

where $\varphi$ adds the identity along $V_{G}^{H}$. There is a homotopy equivalence

$$
G L_{H}\left(\tau_{x}^{H} \oplus V^{H}, \xi_{x}^{H} \oplus V^{H}\right) \simeq O\left(\operatorname{dim} \tau_{x}^{H}+\operatorname{dim} V^{H}\right) .
$$

Thus, $\varphi$ is $\left(\operatorname{dim} \tau_{x}^{H}+\operatorname{dim} V^{G}-1\right)$-connected. Since $\operatorname{dim} \tau_{x}^{H}+\operatorname{dim} V^{G}-1 \geqq$ $\operatorname{dim} X$, stated homotopy group vanishes, consequently the obstruction vanishes. Q.E.D.

If $U, V$, and $W$ are real $G$-modules then let $G L_{G}(U, V, W)$ be the space of all $G$-linear isomorphisms $b: U \oplus W \rightarrow Y \oplus W$ having the form

$$
\left(\begin{array}{cc}
c & d \\
0 & i d_{W}
\end{array}\right) \quad(c: U \rightarrow V, d: U \rightarrow W) .
$$

The subspace $D L_{G}(U, V, W)$ of $G L_{G}(U, V, W)$ is defined to be the space consisting of all $b: U \oplus W \rightarrow V \oplus W$ having the form

$$
\left(\begin{array}{cc}
c & d \\
0 & i d_{W}
\end{array}\right) \quad(c: U \rightarrow V) .
$$

It is clear that the canonical inclusion $D L_{G}(U, V, W) \rightarrow G L_{G}(U, V, W)$ is a homotopy equivalence.

Proof of Theorem 3.5. A required $G$-regular homotopy $b_{t}=b_{X, t}$ is constructed by the cell-by-cell construction as usual. Thus it suffices to prove Theorem 3.5 in a special case where $X=A \cup\left(G / H \times D^{i}\right)$. As in the previous proof, let $Y=\operatorname{Res}_{N}^{G} A \cup\left(N / H \times D^{i}\right)\left(N=N_{G}(H)\right)$. Then it suffices to find an $N$-regular homotopy (relative to $\left.\operatorname{Res}_{N}^{G} A\right) b_{Y, t}:\left.b\right|_{Y} \sim b_{Y}^{\prime}(t \in[0,1])$ such that $b_{Y}^{\prime}$ satisfies the $\left.\operatorname{Res}_{N}^{G} \eta\right|_{Y}$-normal condition. Fix a point $x \in H / H \times \operatorname{Int}\left(D^{i}\right)$. Then $\tau_{x} \oplus \eta_{x}$ is the direct sum $\tau_{x}^{H} \oplus \tau_{x H} \oplus \eta_{x}^{H} \oplus \eta_{x H}$ as real $N$-modules. Required $b_{Y, t}$ must satisfy $\left.b_{Y, t}\right|_{A}=\left.b\right|_{A}$ for all $t \in[0,1]$. Thus set $b_{A, t}=\left.b\right|_{A}$. The obstruction to extending $b_{A, t}$ to $b_{Y, t}:\left.b\right|_{Y} \sim b_{Y}^{\prime}(t \in[0,1])$ over $Y$ such that $b_{Y}^{\prime}$ satisfies the $\left.\operatorname{Res}_{N}^{G} \eta\right|_{Y}$-normal condition lies in $\pi_{i}(\varphi)$ for the canonical inclusion

$$
\begin{aligned}
\varphi: G L_{H}\left(\tau_{x}^{H} \oplus \eta_{x}^{H}, \xi_{x}^{H} \oplus \eta_{x}^{H}\right) \times D L_{H}\left(\tau_{x H}, \xi_{x H}, \eta_{x H}\right) \\
\quad \rightarrow G L_{H}\left(\tau_{x}^{H} \oplus \eta_{x}^{H}, \xi_{x}^{H} \oplus \eta_{x}^{H}\right) \times G L_{H}\left(\tau_{x H}, \xi_{x H}, \eta_{x H}\right)
\end{aligned}
$$

Since $\varphi$ is a homotopy equivalence, the stated homotopy group vanishes, hence the obstruction is trivial. This proves Theorem 3.5 in the case $X=$ $A \cup\left(G / H \times D^{i}\right)$. The general case can be proved by induction on sub-G-complexes of $X$. Q.E.D. 


\section{Acknowledgements}

I would record here my acknowledgements to the referee for his cordially reading the manuscript and correcting spellings and types.

\section{References}

[BMo1] Bak, A. and Morimoto, M., Equivariant surgery and applications, Proc. Conf. on Topology in Hawaii 1991 (ed. by K. H. Dovermann), 12-25, World Scientific Pupl., Singapore, 1992.

[BMo2] — , Equivariant surgery on compact manifolds with half dimensional singular sets, preprint, 1992.

[tD1] tom Dieck, T., Transformation Groups and Representation Theory, Lect. Note. in Math., 766, Springer, Berlin, Heidelberg and New York, 1979.

[tD2] - Transformation Groups, de Gruyter Studies in Math., 8, Walter de Gruyter, Berlin and New York, 1987.

[Dr] Dress, A., A characterization of solvable groups, Math. Z., 110 (1969), 213-217.

[LüMa] Lück, W. and Madsen, I., Equivariant L-theory I, Math. Z., 203 (1990), 503-526.

[LaMo] Laitinen, E. and Morimoto, M., Finite groups with smooth one fixed point actions on spheres, Reports Dept. Math., Univ. of Helsinki, no. 25 (1993).

[LaMoPa] Laitinen, E., Morimoto, M. and Pwałowski, K., Smooth actions of finite nonsolvable groups on spheres, Reports Dept. Math., Univ. of Helsinki, no. 12 (1992).

[Mo1] Morimoto, M., On one fixed point actions on spheres, Proc. Japan Acad., 63A (1987), 95-97.

[Mo2] - Most of the standard spheres have smooth one fixed point actions of $A_{5}$, Transformation Groups (ed. by K. Kawakubo), Lect. Note. in Math., 1375, 240258, Springer, Berlin, Heidelberg and New York, 1989.

[Mo3] — Most standard spheres have smooth one fixed point actions of $A_{5}$ II, $K$ Theory, 4 (1991), 289-302.

[Mo4] — - Positioning map, equivariant surgery obstruction, and applications, RIMS Kokyuroku, 793 (1992), 75-93, Res. Inst. Math. Sci., Kyoto Univ., Kyoto.

[MoU] Morimoto, M. and Uno, K., Remarks on one fixed point $A_{5}$-actions on homology spheres, Algebraic Topology Poznań 1989 (ed. by S. Jackowski, B. Oliver, and K. Pawałowski), Lect. Note. in Math., 1478, 337-364, Springer, Berlin, Heidelberg and New York, 1991.

[O] Oliver, R., Fixed-point sets of group actions on finite acyclic complexes, Comment. Math. Helvetici, 50 (1975), 155-177.

[Pe1] Petrie, T., Pseudoequivalences of G-manifolds, Algebraic and Geometric Topology (ed. by R. J. Milgram), Proc. Sympo. in Pure Math. (Amer. Math. Soc.), 32 (1978), $169-210$.

[Pe2] — $-G$ Surgery I-A survey, Algebraic and Geometric Topology (ed. by K. C. Millett), Lect. Note. in Math., 664, 197-242, Springer, Berlin, Heidelberg and New York, 1978.

[Pe3] —, One fixed point actions on spheres I, Adv. Math., 46 (1982), 3-14.

[PeR] Petrie, T. and Randall, J. D., Transformation Groups on Manifolds, Marcel Dekker, New York, 1984. 
